\title{
Nonlinear response of Black Sea pelagic fish stocks to over-exploitation
}

\author{
Temel Oguz* \\ Institute of Marine Sciences, Middle East Technical University, PO Box 28, Erdemli 33731, Mersin, Turkey
}

\begin{abstract}
A 3-trophic level prey-predator model investigated the underlying nonlinear dynamics governing long-term (1960 to 1999) changes in pelagic fish stocks in the Black Sea. The model first explored the steady state dynamics under various combinations of consumption, harvesting and mortality rate values, and identified the critical parameters and their ranges that control equilibrium characteristics of fish stocks in the pristine state of the ecosystem. This knowledge was then used to describe progression of the stocks under temporally varying harvesting regimes. Although idealized within the structure of the model, the simulations reproduced empirical observations reasonably well. The model possesses different single equilibrium solutions during different phases of the system and associated regime shift dynamics. The late 1960s represented the disruption period of heavilyexploited top predator stock and the successive proliferation of weakly-exploited small and medium pelagic stocks. The high stock regime of small pelagics persisted for a decade and then rebounded back and forth between the low and high abundance regimes. The 1990s ecosystem represented a gradual switch of small pelagics to a high stock regime, whereas other groups preserved their low stock regimes. Simulations further explored optimum harvesting conditions for balanced stocks of small and medium pelagics by the end of next decade. The present study highlights how a simple model, when carefully tuned, may provide detailed information on fish stock dynamics and realistically reproduce the empirical observations. The model also illustrates the value of a minimalist deterministic approach for multi-species fishery management strategy development.
\end{abstract}

KEY WORDS: Black Sea · Pelagic fish populations $\cdot$ Prey-predator model $\cdot$ Abrupt transitions · Quasistable states · Over-exploitation

Resale or republication not permitted without written consent of the publisher

\section{INTRODUCTION}

An extensive body of literature documents dramatic global changes in marine food web structures and functioning under the influence of climatic or humaninduced perturbations. Overfishing is one of the serious ecological concerns. Many of the world's fish stocks, especially large predatory fishes, have been severely depleted within the past $50 \mathrm{yr}$ (Hutchings 2000, Jackson et al. 2001, Myers \& Worm 2003). In intensively fished ecosystems, decline in the abundance of top predatory fish populations has shifted fisheries to target species at lower trophic levels (Pauly et al. 1998). The Black Sea is no exception. Successive over-exploitation of fish stocks (i.e. 'fishing down the food web') together with intense eutrophication, strong decadal-scale climatic cooling/warming, and population outbursts of opportunistic species and gelatinous carnivores concomitantly introduced stresses on the ecosystem far more severe than those encountered in many other semi-enclosed, marginal and shelf seas within the second half of the previous century (Zaitsev \& Mamaev 1997, Gücü 2002, Kideys 2002, Daskalov 2003, Bilio \& Niermann 2004, Oguz, 2005a, Oguz 2005b, Oguz et al. 2006). Utilizing long-term ecological data, Oguz \& Gilbert (2007) recently diagnosed sharp transitions between quasi-stable states of various ecological properties during the reorganisation of an ecosystem under synchronous climatic and anthropogenic forcing. They noted a switch from a large 
predatory fish to a small planktivore fish-controlled system in the early 1970 s, the transition to a gelatinous-controlled system at the end of the $1980 \mathrm{~s}$, and to a small planktivore-controlled system again at the end of the 1990s.

Daskalov (2002) and Oguz \& Gilbert (2007) further noted the inevitable role of trophic cascade process in food web dynamics during times of marked changes in higher trophic level structure in response to excessive and continual harvesting of stocks over different phases of the recent past. Therefore, quantitative understanding of likely responses of the pelagic fish stocks to over-exploitation constitutes one of the key issues for gaining a deeper insight into the mechanisms that govern the long-term organisation of the ecosystem studied. Here, I use a simple deterministic model to explore underlying nonlinear dynamics associated with the long-term stock variations under differing harvesting regimes.

To date, simple prey-predator type population dynamic models dealing with multiple states have generally focused on the equilibrium (i.e. steady-state) properties of fish and plankton populations under different parameter ranges and for various functional representations of consumption and predation mortality mechanisms (May et al. 1979, Steele \& Henderson 1984, Hastings \& Powell 1991, Abrams \& Roth 1994, Spencer \& Collie 1995, Edwards \& Yool 2000, Scheffer et al. 2000, Kemp et al. 2001, Lima et al. 2002, Gibson et al. 2005, Morozov et al. 2005). In a rather idealized way, some models have incorporated impacts of stochastic climatic variability on long-term fish population fluctuations (e.g. Steele \& Henderson 1984, Spencer \& Collie 1995, Collie et al. 2004). The present study extends these efforts to testing the capability of a minimalist model in the simulation of multi-decadal behaviours of pelagic fish populations within a heavily exploited marine environment (i.e. the Black Sea). The subsequent sections provide: (1) a qualitative interpretation of the available catch data to set a basis for the modelling studies; (2) a brief overview of the regime shift concept in ecological systems; (3) equilibrium analyses of the model for elucidating the mechanisms controlling abrupt stock changes and identifying the optimum parameter ranges that are critical to model dynamics; and (4) simulations of long term (1960 to 1999) stock and catch variations and possible stock developments under different harvesting scenarios in the forthcoming decade.

\section{Interpretation of catch observations}

Catch observations are the only available systematic long-term data from which to make inferences on the fate of Black Sea fish stocks during the recent past. The fact that the fishing effort was quite high during the 1970s and 1980s (Gücü 2002) justifies the link between the catch and stock variations, and thus to develop a conceptual view that forms a basis for the present modelling studies. The data set (Fig. 1), retrieved from www.seaaroundus.org/lme/lme.aspx, comprises the total annual landings of small pelagics (anchovy Engraulis encrasicolus, sprat Sprattus sprattus, horse mackerel Trachurus spp., Pontic shad Alosa pontica); of medium size pelagics (Atlantic bonito Sarda sarda, mackerel Scomber spp., blue fish Pomatomus saltator); larger pelagics $(>90 \mathrm{~cm})$; and the total annual landings of small and medium demersals (turbot Scopthalmus rhombus, red mullet Mullus barbatus, whiting Merlangius $\mathrm{m}$. euxinus). The data set also includes the annual dolphin catches by the former Soviet Union countries prior to the harvest ban in 1966 (Zemsky 1996), and by Turkey that continued extensively into the 1970s (Yel et al. 1996).

The first phase in the data covers the 1950s and the early 1960s. It is characterized by low catches of small and medium pelagics and relatively high catches of dolphins and demersal fishes (Fig. 1). Large pelagic fish catches, on the other hand, maintained a steady level around $20 \mathrm{kt}$ throughout the study period. The catch data likely reflect the prevalence of low stocks of large pelagics under intense harvesting and thus their weak predation control on the food web. This system appears to suggest domination of the higher trophic level by large predatory and demersal fish stocks relative to small and medium pelagics. The total catch of the former group varied between 100 and $200 \mathrm{kt}$, whereas that of the others was below $200 \mathrm{kt}$ (Fig. 2).

Dolphin catches declined dramatically by the mid1960. This was accompanied by large reductions in demersal fish catches. Their total low catch level $(<50 \mathrm{kt})$ in the subsequent decades implies a continuous high fishing effort on these stocks. In contrast, first

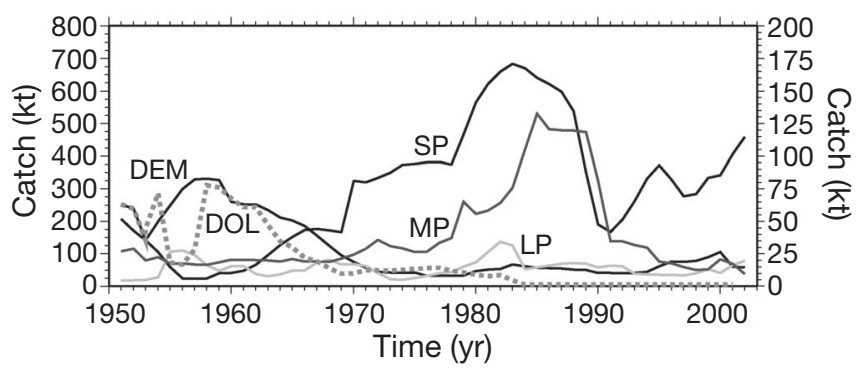

Fig. 1. Long-term catch variations of dolphins (DOL), demersals (DEM), large pelagics (LP), medium pelagics (MP), and small pelagics (SP) from 1950 to 2003. The original data have been smoothed using a 5 point Gaussian filter. The abscissa on the left shows catch values for the small pelagics group, and on the right for the other groups 


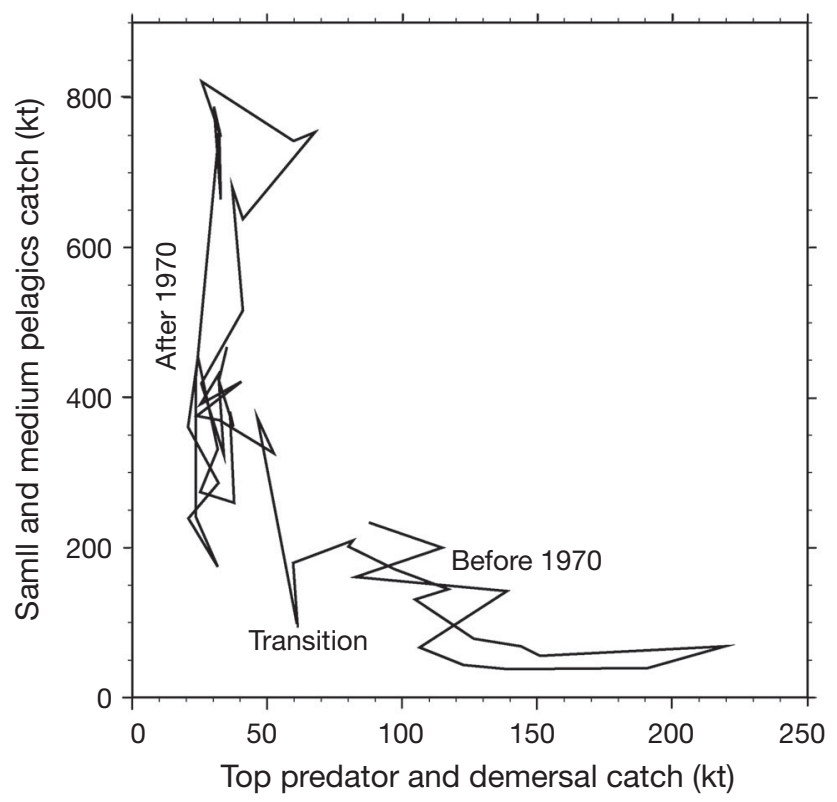

Fig. 2. The sum of top predator (dolphins and large pelagics) and demersal catches versus the total catch of small and medium pelagics, demonstrating 2 distinct modes in the Black Sea fish populations before and after 1970

the small pelagics catch and then the medium pelagics catch increased in the 1980s up to a total of $800 \mathrm{kt} \mathrm{yr}^{-1}$ (Fig. 2), primarily in response to the rapid expansion of Turkish fishing fleets (Gücü 2002). This period therefore should have been able to sustain high stocks of faster-growing species of small and medium pelagics following declines of large predators and demersals (i.e. reduction of original top-down control). Interestingly, similar shifts have been observed on a global scale over the same time period (Myers \& Worm 2003).

The catches of small and medium pelagics declined dramatically and concomitantly at the end of the 1980s. In particular, anchovy populations that were the most abundant fish stocks of the 1980s collapsed rapidly from more than $800 \mathrm{kt}$ to $200 \mathrm{kt}$ (Ivanov \& Panayotova 2001, Daskalov 2002). This phase, however, lasted for only $4 \mathrm{yr}$ and the small pelagics catch started increasing gradually from 1993 up to about $400 \mathrm{kt}$ during the early 2000s. The medium, large predatory and demersal fish catches, on the other hand, persisted at their low background levels during this period.

The catch data may have the following implications with regard to the reorganization of stocks within the last 50 yr: (1) a major shift from a demersal and top predator-dominated system to a small and medium pelagics-dominated system at the end of the 1960s; (2) the existence of 2 successive quasi-persistent low and high stock regimes of the small plus medium pelagics groups and the top predator plus demersal groups (Fig. 2), each lasting for approximately 2 decades between 1950 and 1990; (3) a transition towards a high stock regime of small pelagics during the 1990s at the expense of low stock regimes of medium and large pelagic predators; and (4) 2 distinct short-term transitional periods with lowest stocks of all populations at the end of the 1960s and the end of the 1980s. The model simulations presented in the subsequent sections seek a quantitative support for these assertions.

\section{An overview of regime shifts in ecological systems}

One of the objectives of the present study was to identify the mechanisms responsible for marked changes (i.e. regime shifts) that likely occurred in the Black Sea fish stocks during the second half of the last century. A brief overview of the regime shift concept is therefore appropriate here for the sake of completeness. The ecological configuration of species assemblages may alternate between stable states (also referred to as regimes) when an ecosystem loses its internal resilience under strong environmental stressors (both natural and human-induced), and subsequently some internal and/or external properties of the ecosystem reach critical thresholds (May 1977, Scheffer et al. 2001, Petraitis \& Dudgeon 2004). Transitions between the stable states occur in 2 different forms depending on the equilibrium characteristics of the system. In the case of multiple equilibria, the strongly nonlinear systems possess alternative stable states (e.g. low and high stock regimes) for a particular set of internal or external conditions (e.g. fishing mortality rate, temperature). The properties alternate their states abruptly via forward and backward transitions at 2 different thresholds (c.f. Fig. 2 in Petraitis \& Dugdeon 2004). The transitions are referred to as 'discontinuous' regime shifts and the phenomenon is called 'hysteresis' (Scheffer et al. 2001). The George's Bank (North-West Atlantic) haddock stock variations that resulted from marked changes in harvesting rates (Collie et al. 2004) fall into this category. Discontinuous regime shifts impose strong constraints on reversibility of the states. They are traced by abrupt changes in the time series data.

Ecosystems are more often characterized by a single equilibrium in which a stable state characterized by specific internal and external conditions may alternate to a new stable state as conditions change (c.f. Fig. 3 in Petraitis \& Dugdeon 2004). The states are therefore identified by 2 different ranges of conditions on both sides of the thresholds (instead of the same range between 2 different thresholds in discontinuous shifts). They resemble sinusoidal-type fluctuations in the time series data as in the case of low frequency, multi- 
decadal scale, climate-driven fluctuations observed in open ocean fish populations (de Young et al. 2004). A well-known example is the series of successive transitions between a warm 'sardine regime' and a cool 'anchovy regime' in the Pacific Ocean since the early 1950s (Chavez et al. 2003). Following Scheffer et al. (2001) and Collie et al. (2004), they are referred to as 'smooth' regime shifts here. The discontinuous and linear shifts represent 2 end-members of nonlinear bifurcations, depending on the degree of nonlinearity of the system. Scheffer et al. (2001) and Scheffer \& Carpenter (2003) provide the theoretical framework of regime shifts, as well as some case studies from marine and aquatic ecosystems.

The definitions of regime shifts given above formally apply to systems controlled by one variable (e.g. the consumption rate or harvesting rate). In reality, several internal and/or external factors often impose simultaneous controls on ecological systems, and therefore make the regime shift interpretations ambiguous in terms of identification of the threshold values and the equilibrium characteristics (single or multiple equilibrium). As described in the following sections, this ambiguity also arose in the present study.

\section{METHODS}

Model formulation. The model comprises the small pelagics (resource or prey), medium pelagics (consumer) and large pelagics (top predator) groups; the latter involving both large pelagic fish and dolphins. The small pelagics group grows using resources provided by the lower trophic level, and is consumed by both the medium pelagics and top predator groups. As the top predator and consumer groups compete for a single resource, the predator group feeds on the consumer group as well. The model resembles an intra-guild omnivory system, such as phytoplankton (resource), microzooplankton (consumer) and mesozooplankton (predator). For simplicity, demersal stock variations and their interactions with small pelagics were not explicitly taken into account, because of their negligible role on the Black Sea fishery after the 1960s. All groups were subjected to a linear harvesting, and the system was closed by the quadratic mortality terms for the top predator and medium pelagics groups. A higher degree of complexity introduced by nonlinear couplings between the consumption, mortality and harvesting mechanisms of these 3 groups make the model more sophisticated than the earlier one preyone predator models (e.g. Spencer \& Collie 1995) and 3-trophic level food-chain models (Hastings \& Powel 1991, Abrams \& Roth 1994). In essence, the model complexity conceptually resembles that presented by
May et al. (1979) who investigated the dynamic response of the Antarctic ecosystem to changes in harvesting regimes on different fish species. The governing equations, in their dimensional form, are expressed by:

$$
\begin{aligned}
\frac{\mathrm{d} S_{1}}{\mathrm{~d} t^{*}}= & R_{1} S_{1}\left(1-\frac{S_{1}}{K_{1}}\right)- \\
& R_{2} S_{2} \frac{S_{1}^{2}}{K_{2}^{2}+S_{1}^{2}}-R_{3} S_{3} \frac{a_{1} S_{1}^{2}}{K_{3}^{2}+a_{1} S_{1}^{2}+a_{2} S_{2}^{2}}-f_{1}^{*} S_{1} \\
\frac{\mathrm{d} S_{2}}{\mathrm{~d} t^{*}}= & \varepsilon_{2} R_{2} S_{2} \frac{S_{1}^{2}}{K_{2}^{2}+S_{1}^{2}}- \\
& R_{3} S_{3} \frac{a_{2} S_{2}^{2}}{K_{3}^{2}+a_{1} S_{1}^{2}+a_{2} S_{2}^{2}}-D_{2} S_{2}^{2}-f_{2}^{*} S_{2} \\
\frac{\mathrm{d} S_{3}}{\mathrm{~d} t^{*}}= & \varepsilon_{3} R_{3} S_{3} \frac{a_{1} S_{1}^{2}+a_{2} S_{2}^{2}}{K_{3}^{2}+a_{1} S_{1}^{2}+a_{2} S_{2}^{2}}-D_{3} S_{3}^{2}-f_{3}^{*} S_{3}
\end{aligned}
$$

In Eqs. (1a), (1b) \& (1c), the operator $\mathrm{d} / \mathrm{d} t^{*}$ represents the time derivative; $S_{1}, S_{2}, S_{3}$ denote, respectively, small, medium and top predator pelagic fish abundances; $R_{1}$ defines growth rate of small pelagics; $K_{1}$ is their carrying capacity; $R_{3}$ is the consumption rate of the top predator group on small and medium pelagics' populations with respective percent feeding preference rates of $a_{1}$ and $a_{2} ; R_{2}$ is consumption rate of medium pelagics on small pelagics population; $K_{2}$ and $K_{3}$ are prey abundances, where consumption rates are half their maximum values; $\varepsilon_{2}, \varepsilon_{3}$ are food conversion efficiencies (i.e. the proportion of food assimilated by consumers); $f_{1}{ }^{*}, f_{2}{ }^{*}$ and $f_{3}{ }^{*}$ are harvesting rates, and $D_{2}$ and $D_{3}$ denote mortality rates.

In general, the quadratic mortality term used in the consumer and predator equations parameterizes the consumption that was not explicitly incorporated in the models, and the natural mortality is often considered a part of this loss term. Its nonlinear form, which in biological sense implies a density dependent mortality, is preferred to the linear representation in order to suppress unstable oscillations in the models and to stabilise solutions (e.g. Edwards \& Yool 2000). The density dependent mortality $\left(d_{k} S_{k \prime}, k=2,3\right.$; see Eqs. 1b,c), however, goes to zero as the top predator stock vanishes. A better choice may be to use both density dependent and independent terms (Caswell \& Neubert 1998). The quadratic mortality term is omitted in Eq. (1a), since the predation mortality by the medium pelagics and top predator groups have already been explicitly taken into account in the model. On the other hand, because of the presence of the linear fishing mortality term in Eq. (1a), a separate linear natural mortality loss term would be redundant within the framework of this simplified 
model. The natural mortality was simply assumed to be a fraction of the linear loss term. Low rates of natural mortality of adult fishes, which form the main stocks for fishery, justify this approximation.

The model incorporates neither age-structure of populations nor seasonal variability and spatial structure. The model was further simplified by assuming no active biological and physical processes regulating population sizes. The lower trophic structure was not explicitly represented, but its contribution was parameterized by the carrying capacity in the logistic growth term. Based on these simplifications, the model offers the first-order dynamical behaviour of the system and forms a basis for building up more sophisticated models that allow complex interactions among different trophic levels. Equations were solved by the 4th-order Runge-Kutta numerical algorithm with a time step of $60 \mathrm{~s}$. A time step up to $1 \mathrm{~h}$, however, gave comparable results.

Nonlinear dynamics of these systems are well known. The combination of a logistic growth functional form, together with sigmoidal Type III consumption, the quadratic natural and/or unparameterized predator mortality and the linear fishing mortality functions imply either single high or low stable equilibrium of stocks, or 3 equilibria (two stable equilibria separated by an unstable equilibrium) for different combinations of parameter values. In the case of multiple equilibria, a slight change in one of the parameters can cause bifurcation of prey-predator stocks to their alternative domains of attraction (May et al. 1979, Steele \& Henderson 1984, Spencer \& Collie 1995, Caswell \& Neubert 1998, Edwards \& Yool 2000, Scheffer et al. 2000, Kemp et al. 2001, Lima et al. 2002, Gibson et al. 2005, Morozov et al. 2005). The system may also provide single equilibrium, limit cycles and chaotic fluctuations for some parameter ranges (Hast-

Table 1. Nondimensional parameters and their values used in the simulations. See 'Methods' for definitions of parameters

\begin{tabular}{|lcc|}
\hline $\begin{array}{l}\text { Non- } \\
\text { dimensional } \\
\text { parameters }\end{array}$ & $\begin{array}{c}\text { Dimensional } \\
\text { parameters }\end{array}$ & $\begin{array}{c}\text { Range of nondimensional } \\
\text { parameter values used } \\
\text { in the simulations }\end{array}$ \\
\hline$K$ & $K_{1} / K_{2}$ & $40-50^{\mathrm{a}}$ \\
$r_{2}$ & $R_{2} / R_{1}$ & $0.5-0.7$ \\
$r_{3}$ & $R_{3} / R_{1}$ & 0.7 \\
$d_{2}$ & $D_{2} K_{3} / R_{1}$ & $0.0-0.01$ \\
$d_{3}$ & $D_{3} K_{3} / R_{1}$ & $0.01-0.04$ \\
$f_{i}$ & $f_{i}^{*} / R_{1}$ & $0.25-0.90^{\mathrm{a}}$ \\
$\gamma$ & $K_{2} / K_{3}$ & 2.5 \\
$F_{1}$ & $S_{1} / K_{2}$ & Predicted \\
$F_{2}$ & $S_{2} / K_{3}$ & Predicted \\
$F_{3}$ & $S_{3} / K_{3}$ & Predicted \\
aTime dependent & \\
\hline
\end{tabular}

ings \& Powell 1991, Abrams \& Roth 1994, Caswell \& Neubert 1998).

In order to make the analysis more tractable, Eqs. (1a), (1b) and (1c) were made nondimensional by scaling $S_{1}$ with $K_{2}, S_{2}$ and $S_{3}$ with $K_{3}$, time $t$ with $R_{1}^{-1}$, and other dimensional parameters as shown in Table 1. Eqs. (1a) to (1c) then transform to:

$$
\begin{aligned}
\frac{\mathrm{d} F_{1}}{\mathrm{~d} t}= & F_{1}\left(1-\frac{F_{1}}{K}\right)-\frac{r_{2}}{\gamma} F_{2} \frac{F_{1}^{2}}{1+F_{1}^{2}}- \\
& \frac{r_{3}}{\gamma} F_{3} \frac{a_{1} \gamma^{2} F_{1}^{2}}{1+a_{1} \gamma^{2} F_{1}^{2}+a_{2} F_{2}^{2}}-f_{1} F_{1} \\
\frac{\mathrm{d} F_{2}}{\mathrm{~d} t}= & \varepsilon_{2} r_{2} F_{2} \frac{F_{1}^{2}}{1+F_{1}^{2}}- \\
& r_{3} F_{3} \frac{a_{2} F_{2}^{2}}{1+a_{1} \gamma^{2} F_{1}^{2}+a_{2} F_{2}^{2}}-d_{2} F_{2}^{2}-f_{2} F_{2} \\
\frac{\mathrm{d} F_{3}}{\mathrm{~d} t}= & \varepsilon_{2} r_{2} F_{2} \frac{a_{1} \gamma^{2} F_{1}^{2}+a_{2} F_{2}^{2}}{1+a_{1} \gamma^{2} F_{1}^{2}+a_{2} F_{2}^{2}}-d_{3} F_{3}^{2}-f_{3} F_{3}
\end{aligned}
$$

The values for dimensional growth rate and carrying capacity of small pelagics were taken from the available literature as $R_{1}=0.9 \mathrm{y}^{-1}, K_{1}=2000 \mathrm{kt}$; this approximately corresponds to twice the maximum observed stock value of small pelagics. The nondimensionalisation of equations introduced a new parameter $\gamma=K_{2} / K_{3}$ that defines the ratio of half saturation constants of consumer and top predator abundances. Thus, $\gamma$ sets the value of $K_{3}$ relative to $K_{2}$ in the model. The stocks were scaled by $K_{2}$ and $K_{3}$ and the value of $K_{2}$ was set to $50 \mathrm{kt}$, whereas the value of $K_{3}$ depended on the choice of $\gamma$. The feeding preference rate of top predator and medium pelagics groups were $a_{1}=0.7$ and $a_{2}=0.3$, respectively. This setting suggests more efficient prey consumption of the top predator group on small pelagics in comparison with the medium pelagics groups. The food conversion efficiencies $\varepsilon_{2}, \varepsilon_{3}$ were taken as 0.7 . These parameter values were kept fixed in all simulations, because the model is not critically sensitive to their changes under their expected range of variations for the Black Sea. Other parameter values, particularly the parameter $\gamma$ and the nondimensional consumption rates $r_{2}$ and $r_{3}$, were not precisely known and preliminary sensitivity experiments suggested their critical importance in structuring the long-term observed stock and catch variations. Their optimum values that reproduced the observed stocks and catches during different phases of the system were assessed by the equilibrium analyses of the model, instead of choosing them from a broad range of observed values reported for different species. The alternative and/or complementary approach would be their estimation with a 
nonlinear parameter optimization technique in the case of a more complete data set.

Equilibrium analyses. I refer to Fig. 2 to reiterate the hypothesis put forward on the existence of 2 distinctly different regimes of the fish stocks before and after 1970. The existence and equilibrium characteristics of these 2 quasi-steady phases of the system were examined under various combinations of the harvesting, predation and mortality parameter values. For this purpose, an extensive set of experiments were performed in silico by systematically altering the parameter values within their dynamically feasible ranges. A sample from this set that specifically applies to the Black Sea conditions is presented below. More general aspects of the prey-predator dynamics given by this relatively complex 3-trophic level model will be reported elsewhere.

\section{RESULTS}

\section{Response of the system to the ratio of half saturation constants}

I first focus on the response of the pristine system (1950s and 1960s) to the parameter $\gamma$. The equilibrium solutions were sought for its values changing between 0.5 and 5.0 at an increment of 0.5 for different choices of $r_{2}$ and $r_{3}$ in the ranges given in Table 1 . The first solution for $\gamma=0.5$ was obtained from an initial state representative of the pristine conditions, with $F_{1}=F_{2}=3.0$ (the low stock states of small and medium pelagics groups; 150 and $60 \mathrm{kt}$, respectively) and $F_{3}=10.0$ (the high stock state of top predator group; $200 \mathrm{kt}$ ), with a model run of $20 \mathrm{yr}$, which was long enough to achieve the steady state solution. Each of the subsequent solutions for other $\gamma$ values was obtained by 20 yr integration of the model using the former steady state solution as initial conditions. Other parameters were chosen as $K=40, f_{1}=0.25$, $f_{2}=f_{3}=0.30, d_{2}=d_{3}=0.01$ (the rest are given above) . The $K$ and $f$ values reflected low harvesting and moderate carrying capacity of the pristine system during the 1950s and 1960s.

The equilibrium solutions were obtained for both increasing and decreasing values, in order to determine whether the system possessed single or multiple equilibrium states. The solutions indicated that the small and medium pelagics stocks were not sensitive to the choice of $\gamma$ up to its value of 4.0, and they permanently resided in their low stock regimes. The stocks flipped however to their high stock regimes when $\gamma \geq 4$.0. The top pelagics changed gradually from the low to high stock regime with increasing $\gamma$ values.
The cases with $\gamma \geq 4.0$ (i.e. $K_{2}=50$ and $K_{3} \leq 12.5$ ) represented a condition in which the prey group had an efficient growth, supplied high resource levels to the consumer and predator groups, and gave rise to high stocks for the entire system. Conversely, the cases with $\gamma \leq 1.0$ (i.e. $K_{2}=50$ and $K_{3} \geq 50$ ) possessed severe food limitation and exhaustion of stock at all trophic levels. The choices of $\gamma$ between 1.0 and 4.0 introduced strong top-down control by top predators on the prey and consumer groups. Among all these combinations, $\gamma=2.5$ revealed top pelagic stock and catch values consistent with the empirical observations during the 1950s and 1960s. This value was therefore adopted for the rest of the analyses.

\section{Response of the system to consumption rates}

The sensitivity of the system to values of $r_{2}$ and $r_{3}$ was determined next in order to identify the range of values that adequately described the dual-mode structure of the observed fish stocks. The equilibrium solutions were obtained for changing the values of $r_{3}$ from zero to 2.0 at an increment of 0.1 for different choices of $r_{2}$. The stock variation of each group versus $r_{3}$, depicted in Fig. 3 for 3 different consumer consumption rates $r_{2}=$ $0.5,0.7,0.9$, respectively, reveals the single equilibrium of the system. Either the small pelagics group or the medium pelagics group, or both, depending on the value of $r_{2}$, lay in a state which was opposite that of the top predator group for any value of $r_{3}$. The transition between the stable states occurred at slightly different threshold values of $r_{3}$ for all groups. For example, for $r_{2}=0.5$, the small pelagics group was in the high stock regime up to $r_{3}=0.7$, then shifted to the low stock regime in the range $0.7<r_{3}<0.9$, and remained stable thereafter with increasing values of $r_{3}$ (Fig. 3a). The top predator group showed reverse characteristics with increasing $r_{3}$ values (Fig. 3c). The medium pelagics group remained permanently in the low stock regime for the entire range of $r_{3}$ (Fig. 3b), suggesting that its consumption rate on small pelagics was not sufficiently high to maintain net growth. With decreasing values of $r_{3}$, the states switched at a slightly lower range of $0.4<$ $r_{3}<0.5$ (Fig. 3a,c). The small and top predator groups therefore acquired both low and high stock regimes within a narrow range of $r_{3}$ (during the transition phases of stocks). The top predator group exhibited limit cycle solutions during the transition, as is evident in the peak at $r_{3}=0.8$ (Fig. $3 \mathrm{c}$ ).

For $r_{2}=0.7$, the structures of small pelagics and top predator stocks resembled those for $r_{2}=0.5$ but, in addition, the medium pelagic stock acquired 2 different stable states on either side of the same threshold range of $r_{3}$. The high stock regime of medium 

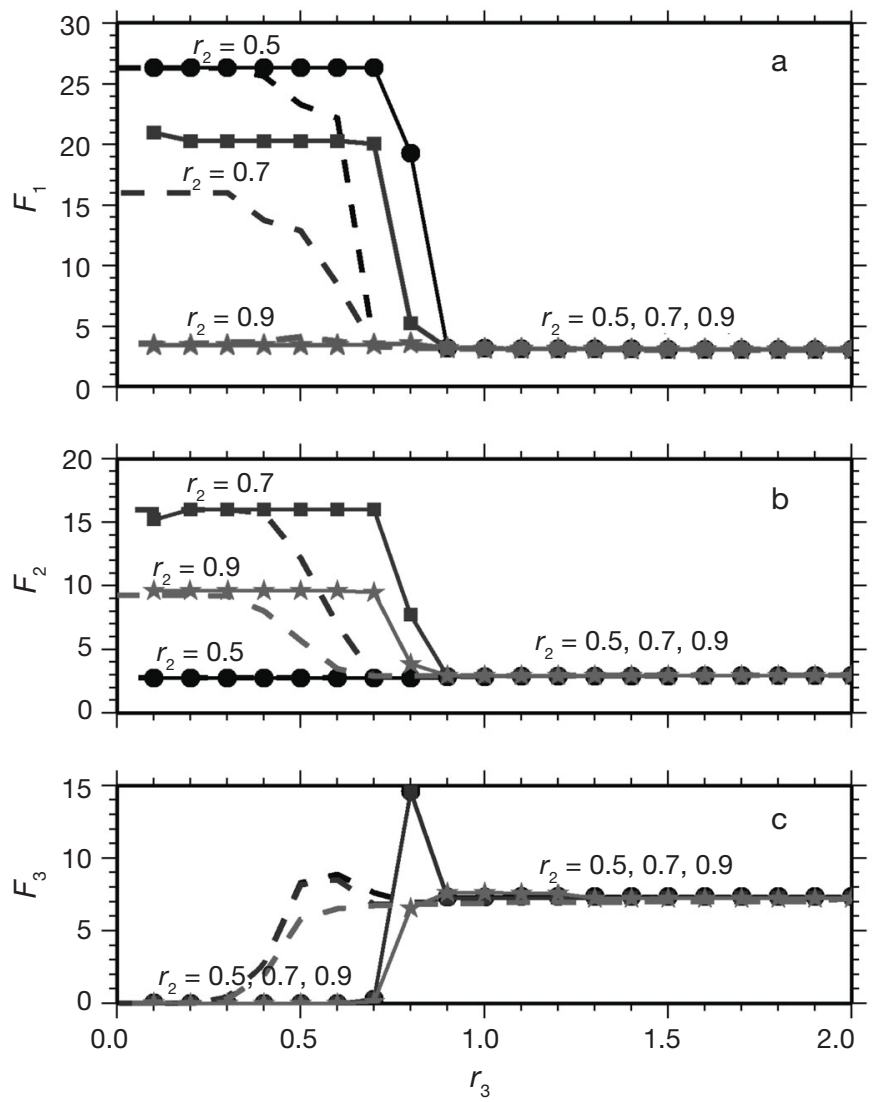

Fig. 3. Relative stock biomass $(F)$ versus top predator consumption rate variations $\left(r_{3}\right)$ obtained from the equilibrium solution of the model for (a) small pelagic, (b) medium pelagic and (c) top predator groups for both increasing (continuous lines) and decreasing (broken lines) values of $r_{3}$ and different choices of the medium pelagic consumption rate: $r_{2}=0.5(\mathbf{)}$, $r_{2}=0.7(\boldsymbol{\square}), r_{2}=0.9(\star)$. The stock curves for $r_{2}>0.9$ are the same as those for $r_{2}=0.9$ and therefore are not shown. The peaks shown in (c) correspond to limit cycle solutions of the model. For the sake of clarity, the symbols are not shown for the solutions with decreasing $r_{3}$ values. All parameters are nondimensional (see Table 1)

pelagics occurred at the expense of a reduced small pelagic stock, while the top predator group retained its former stock size. For decreasing $r_{3}$ values, the transition of the medium pelagics stock took place more gradually between $r_{3}=0.7$ and $r_{3}=0.4$. For $r_{2}=0.9$, the small pelagics stock was completely exhausted, irrespective of the value of $r_{3}$, due to a stronger consumption pressure exerted by medium pelagics. The reduction in medium pelagics stock size (in comparison with stock size at $r_{2}=0.7$ ) reflected relatively weaker growth, due to the exhaustion of small pelagics stock under high predation pressure from medium pelagics, and points to a feedback mechanism between the consumption and growth characteristics of the prey-consumer system. The structure of the top predator stock gen- erally remained unchanged during the transition phase, except for weak oscillations. Under the present parameter setting, the equilibrium analysis indicates that the consumption rate values of $r_{3}>0.7$ and $0.5<r_{2}<0.9$ are the most appropriate for modelling the Black Sea prey-consumer-predator system. Beyond these ranges, the solutions are not relevant to the Black Sea.

A closer focus on Fig. 3 may help to further constrain the optimum choices of $r_{2}$ and $r_{3}$. For example, values of $r_{3} \gg 0.7$ stabilised the small and medium pelagic stocks in their low stock regimes and did not permit alternation of states when the system was perturbed by other environmental parameters (e.g. increasing fishing mortality rate of the top predator group). Therefore, the optimum choice of $r_{3}$ should be around 0.7 . The choices of $r_{2}>0.7$ for decreasing $r_{3}$ values drastically reduced the small pelagics stock abundance at the expense of a compensatory increase in the abundance of medium pelagics. The computed small pelagics stock size was $F_{1} \approx 26(1300 \mathrm{kt})$ for $r_{2}=0.5$, and $\approx 16$ (800 kt) for $r_{2}=0.7$ (Fig. 3a). Thus, the choice of $r_{2}$ around 0.6 predicted the small pelagics stock size consistent with the empirical observations ( 1100 kt), and also supported the low stock regime of medium pelagics at the time of abrupt stock increase of small pelagics. The time-dependent simulations described in the next section provide further examples of stock variations using various combinations of $r_{2}$ and $r_{3}$ values between 0.6 and 0.7 .

\section{Response of the system to mortality rates}

The equilibrium characteristics of the first phase of the Black Sea fish stocks were also sensitive to the values of top predator mortality rate $d_{3}$. Higher values of $d_{3}$ resulted in the alternation of states; the top predator stock switched to the low equilibrium state and the small and medium pelagics stocks jumped to their high equilibrium states at progressively lower thresholds of $f_{3}$. They even permanently resided at the high equilibrium state for $d_{3} \geq 0.05$, independently of the value of $f_{3}$. As expected, higher values of $d_{3}$ more effectively reduced the top predator stock when it was in the high stock regime (i.e. at low $f_{3}$ values). The analysis suggests the optimum choices of $d_{3}$ lie between 0.01 and 0.04 .

The model dynamics were altered by the presence/ absence of the medium pelagics' mortality closure term in Eq. (2b). In the absence of this term (i.e. $d_{2}=0$ ), the small and medium pelagics stocks exhibited more dominant low frequency oscillations within the threshold range of $f_{3}$. The period of oscillation was about $31.1 \mathrm{yr}$ for $f_{3}=0.40,26.6 \mathrm{yr}$ for $f_{3}=0.45$, and around 
$20 \mathrm{yr}$ for $f_{3}=0.5$ prior to stabilization of the solution after $30 \mathrm{yr}$ of integration. On the other hand, setting $d_{2}$ to a small value around 0.005 to 0.01 stabilized the solution, and this range was adopted in our simulations. The relatively low values of $d_{2}$ were justified because consumption of the medium pelagics stock by the top predator group had already been included in the model. The present contribution represents mainly the natural mortality that is expected to be a low value for their adult populations.

\section{Response of the system to harvesting rates}

Analysis for the first phase

Having constrained the critical ranges of $r_{2}$ and $r_{3}$, the equilibrium analyses next explored how the first phase of the system would respond to the variations of $f_{3}$. The primary interest was to locate the range of $f_{3}$ values which exhausted the top predator stock and enlarged the others. Keeping the previous parameter settings $\left(\gamma=2.5, K=40, f_{1}=0.25, f_{2}=0.30, d_{2}=d_{3}=0.01\right.$ ) and letting $r_{3}=0.7$, equilibrium solutions were obtained for increasing and decreasing $f_{3}$ values between zero and 1.0, at an increment of 0.05 for different $r_{2}$ values. As in the previous case, the small and medium pelagic stocks responded nonlinearly to the top predator fishing mortality rate variations (Fig. $4 \mathrm{a}, \mathrm{b}$ ). For $f_{3} \leq$ 0.35 , these stocks existed at their low stock regimes, but they flipped to the high stock regime at different stock sizes, depending on the value of $r_{2}$ when $f_{3} \geq 0.4$. The choice of $r_{2}=0.5$ allowed only the small pelagics stock to switch to the high stock regime $\left(F_{1} \approx 28\right.$; $1400 \mathrm{kt}$ ), whereas the medium pelagics stock remained at its former state $\left(F_{2} \approx 3 ; 60 \mathrm{kt}\right)$. The choice of $r_{2} \geq 0.9$ always gave rise to the low (high) equilibrium state of small (medium) pelagics stocks over the entire range of $f_{3}$ (Fig. $4 \mathrm{a}, \mathrm{b}$ ). The choice of $r_{2}=0.7$ switched both the small and medium pelagics stocks to their high stock regimes at the same threshold value of $f_{3}(0.35$ to 0.45$)$ and resulted in a more realistic solution for the Black Sea. Moreover, setting $r_{3} \approx 0.7 \geq r_{2}$ favoured small pelagic dominance relative to medium pelagics at the high equilibrium state of system, as in the empirical observations (Fig. 3). The optimum values of $r_{2}$ that made both small and medium pelagics stocks change their stable states lay between 0.6 and 0.7 . They agree with the former analysis of stock variations as a function of consumption rates.

At first glance, the choice of $r_{3} \geq r_{2}$ contradicts the general view that smaller fish species consume at a higher rate and grow faster than larger species. In the model, the only way that the top predator stock dominated that of medium pelagics was to have compara-
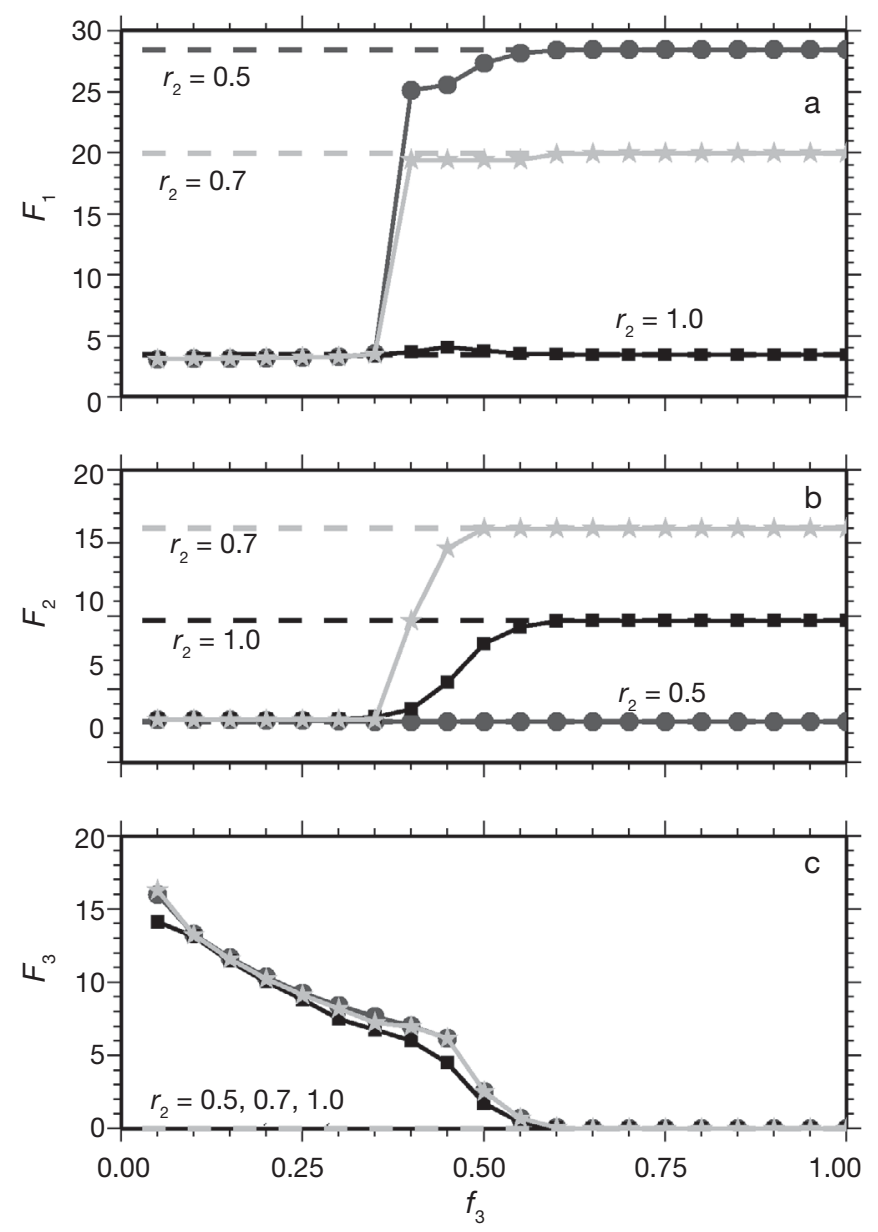

Fig. 4. Relative stock biomass $(F)$ versus top predator fishing mortality rate variations $\left(f_{3}\right)$ obtained from the equilibrium solution of the model for (a) small pelagics, (b) medium pelagics and (c) top predator groups for both increasing (continuous lines) and decreasing (broken lines) values of $f_{3}$ and for different values of the medium pelagics consumption rates: $r_{2}=0.5(\circlearrowleft), r_{2}=0.7(\star)$, and $r_{2}=1.0(\square)$. For the sake of clarity, the symbols are not shown for the solutions with decreasing $f_{3}$ values

ble to or slightly greater effective annual consumption rate of top predators. The choice of $r_{3} \geq r_{2}$ accommodated this effect under low harvesting rate conditions of the first phase. In reality, the top predator group consumed small pelagics for almost the entire year, whereas the medium pelagics group consumed them most effectively for only a certain part of the year when young medium pelagic fish species migrate from the neighboring Aegean and Marmara Seas. Moreover, the stock size of migrating young medium pelagic species is much smaller than their actual annual standing stock sustained in the Black Sea, and was therefore not explicitly incorporated in the model. This simplification was verified by the model simulations. 
Contrary to the nonlinear response to $f_{3}$ by the small and medium pelagics groups, the top predator stock displayed a linear response (c.f. Eq. 1c). It decreased from its maximum values $F_{3} \approx 15$ (300 kt) for increasing $f_{3}$ and vanished at $f_{3} \approx 0.55$ (Fig. $4 \mathrm{c}$ ), which is roughly equal to $\varepsilon_{3} r_{3}$ (i.e. the maximum value of the effective growth rate of the top predator group). The threshold range $f_{3} \approx 0.45$ to 0.55 remained the same across different choices of $d_{3}$ or $f_{1}, f_{2}$.

A striking feature of the steady-state solutions was the existence of both single equilibrium and multiple equilibria of the system for different ranges of $f_{3}$. For $f_{3}>0.4$, the system possessed one stable state for both its increasing and decreasing values. It was, however, characterized by 2 alternative stable states for values of $f_{3}$ between zero and 0.4 for all choices of $r_{2}$. During the decreasing phase of $f_{3}$, the stocks did not shift back to their former states; instead they all resided permanently at their present states. The system thus showed a strong resistance (hysteresis) to the recovery of top pelagic stock and the collapse of other stock groups under decreasing $f_{3}$, as long as the harvesting rates of the small and medium pelagics groups were low $(\sim 0.3)$. This is indeed a desirable fishery management option. The top pelagic stock may, however, recover under different harvesting conditions, an example of which is described further below.

\section{Analysis for the second phase}

The equilibrium analyses were further extended to elucidate the form of stock variations under different combinations of 3 harvesting rates that likely applied for the second phase of long-term stock variations (1970 to 1985) following the first regime shift event. The consumption rates were set to $r_{2}=0.6$ and $r_{3}=0.7$, as deduced from the previous analysis and the other parameters were taken as before. Fig. 5 displays stock variations for both increasing and decreasing values of $f_{1}$ for 3 different choices of $f_{3}(0.3,0.5,0.7)$ and 2 choices of $f_{2}(0.3,0.5)$. Under low harvesting pressure on the medium and top predator pelagics groups $\left(f_{2}=0.3\right.$ and $f_{3}=0.3$ ), the small and medium pelagics stocks resided persistently at their low stock regimes for all choices of $f_{1}$ (Curve 1). They were, however, identified by their high stock regime when $f_{3} \geq 0.5$ and $f_{2}=0.3$ (Curves 2 and 3 ), as shown previously in Fig. 4 a. For $f_{3} \geq 0.5$ and increasing $f_{1}$, the small pelagics stock diminished gradually in the high stock regime, and then more steeply during the transition to the low stock regime at the threshold range of $f_{1}=0.55$ to 0.60 . Increasing $f_{1}$ did not affect stability of the high stock state of medium pelagics up to the threshold value of $f_{1}$. But once the small pelagics stock started decreasing at values of $f_{1}$ beyond
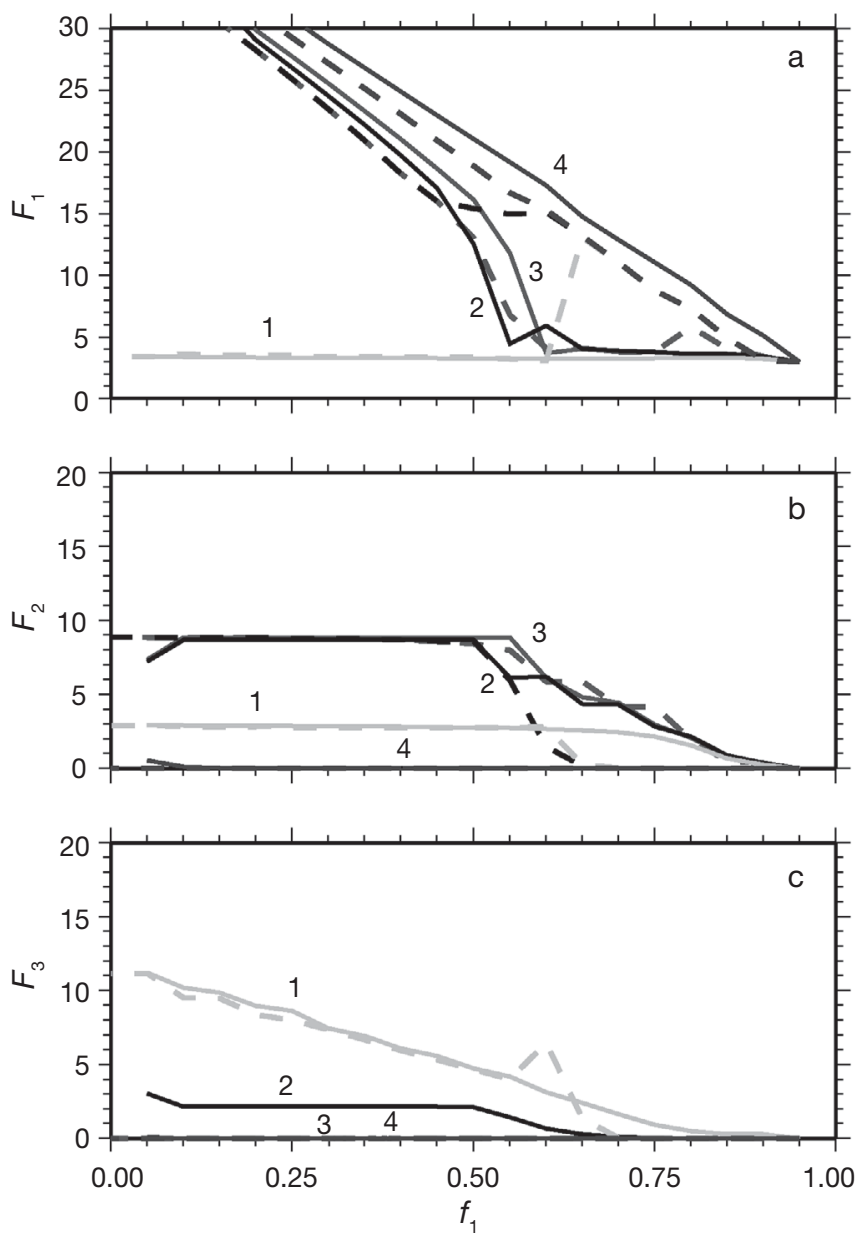

Fig. 5. Relative stock biomass $(F)$ versus small pelagics fishing mortality rate variations $\left(f_{1}\right)$ obtained from the equilibrium solution of the model for (a) small pelagics, (b) medium pelagics, and (c) top predator groups for different choices of the medium pelagics and top predator fishing mortality rates: $f_{2}=$ 0.3 and $f_{3}=0.3$ (Curve 1$), f_{2}=0.3$ and $f_{3}=0.5$ (Curve 2), $f_{2}=0.3$ and $f_{3}=0.7$ (Curve 3), and $f_{2}=0.5$ and $f_{3}=0.7$ (Curve 4). The continuous and broken lines represent stock variations for increasing and decreasing $f_{1}$, respectively

its threshold, the medium pelagic stock also alternated gradually to the low stock regime as its growth became limited due to the food shortage (Fig. 5b). The top predator stock that was in the low stock regime for $f_{3} \geq$ 0.5 was subject to a linear decrease with increasing $f_{1}$, also due to resource limitation (Fig. 5c).

At higher values of $f_{2}$ (e.g. 0.5 and 0.7 ) and $f_{3}$ (e.g. 0.7 ), small pelagics switched from high to low stock regime along a linear trajectory in response to weaker predation pressure from the medium pelagics group (Curve 4 in Fig. 5a). Small pelagics could thus maintain a relatively higher stock size as they were simultaneously harvested, but were placed eventually in the low stock state at $f_{1} \approx 0.8$, together with the other groups (Fig. 5b,c). The entire pelagic stocks collapsed 
at moderate $f_{2}$ and $f_{3}$ values and $f_{1} \geq 0.8$, as observed in the Black Sea at the end of 1980s prior to the recovery of small pelagics stock (see the next section for details).

\section{Analysis for the third phase}

For decreasing $f_{1}$ (as harvesting conditions of the small pelagics group were restored to those before the shift), the stocks generally reverted to their previous states along the same trajectories (see the curves shown by broken lines in Fig. 5). The thresholds of $f_{1}$ for the forward and backward transitions were almost the same within the range of 0.5 to 0.6 , and the transitions are thus classified as 'smooth' regime shifts. For low $f_{2}$ and $f_{3}(\sim 0.3)$, the return trajectory of small pelagics stock (Curve 1), however, exhibited unstable oscillations at high $f_{1}$, but the solutions were eventually stabilised prior to the shift to the high equilibrium state. Similar oscillations also appeared for the case of $f_{2}=0.3$ and $f_{3}=0.5$. The return trajectories of small and medium pelagics followed different paths with respect to their forward trajectories up to the threshold range of $f_{1}$, but merged with the forward trajectories once the stocks alternated the states at lower $f_{1}$ values. As discussed below, the solutions for decreasing $f_{1}$ describe the conditions of stocks recovery after the early 1990s.

The solutions with decreasing $f_{1}$ also displayed an interesting top predator stock recovery case that offered an alternative to the previous no-recovery case under low harvesting conditions of small pelagics $f_{1}$ $\sim 0.3$ (Fig. 4c). The recovery case required initially relatively high $f_{1}$ and $f_{3}$ values $\left(f_{1}>0.6, f_{3} \geq 0.5\right)$ that moved small pelagics to their low stock regime at any value of $f_{2}$ (Curve 1 in Fig. 5a,c). Once small pelagics were settled in the low stock regime and the harvesting rate of top predators was low $\left(f_{3} \approx 0.3\right)$, reduction in $f_{1}$ did not alter the state of small pelagics, but promoted the top predator stock to flip into the high stock regime. Progressively lower $f_{1}$ values gave rise to higher stock size of top predators.

\section{Time-dependent stock variations}

Having identified the optimum ranges of critical model parameters and the underlying dynamics of precipitous stock variations by steady-state analysis, 3 groups of time-dependent simula-
Table 2. Nondimensional parameter values of consumption and natural mortality rates used in 3 long-term simulations of observed stock and catch variations. See 'Methods' for definitions of parameters

\begin{tabular}{|lccccc|}
\hline \multirow{2}{*}{$\begin{array}{l}\text { Simulation } \\
\text { no. }\end{array}$} & \multicolumn{5}{c}{ Nondimensional parameters } \\
\cline { 2 - 5 } & $r_{2}$ & $r_{3}$ & $D_{2}$ & $d_{3}$ \\
\hline 1 & 0.62 & 0.7 & 0.005 & 0.04 \\
2 & 0.65 & 0.6 & 0.007 & 0.04 \\
3 & 0.65 & 0.68 & 0.01 & 0.03 \\
\hline
\end{tabular}

tions are now presented to describe the progression of stocks under temporally varying harvesting conditions. The first group shows how the observed catch variations can be simulated as closely as possible within the framework of this simplified model. The second group highlights a dynamically different stock progression under somewhat different temporal variations of the top predator harvesting rate. The third group of experiments offers alternative stock development scenarios within the next 2 decades.

Simulations of observed stock and catch variations

Parameter setting. Among the large number of prognostic simulations, only 3 that are almost identical and consistent with the available observations are presented here. The values of $r_{i}$ and $d_{i}(i=2,3)$ are given in Table 2, and other parameters were as before: $\gamma=$ $2.5, a_{1}=0.7, a_{2}=0.3, \varepsilon_{1}=\varepsilon_{2}=0.7$. The time integration of the model started from the initial state at 1960 and continued for $40 \mathrm{yr}$ (36 nondimensional time units) until 1999. The initial state was described by low

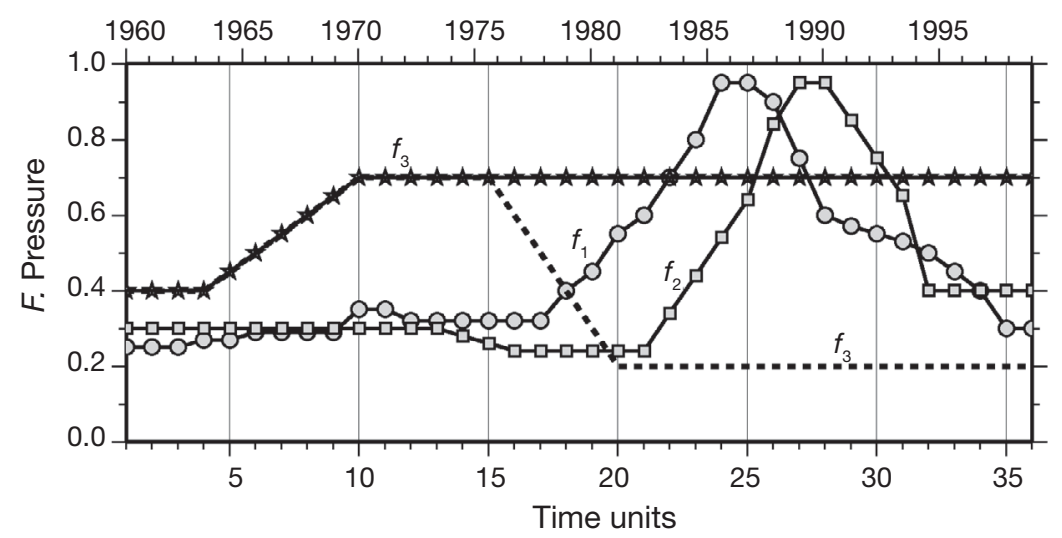

Fig. 6. Temporal variations of fishing mortality rates (F. Pressure) for the small pelagics group $\left(f_{1}, O\right)$, medium pelagics group $\left(f_{2}, \square\right)$, and top predator group $\left(f_{3}, \star\right)$ used in the simulations of observed stock variations. The broken line represents the alternative fishing mortality rate variation for the top predator group used to show possible recovery of the top predator stock 
stocks of the small and medium pelagics groups at $F_{1}=$ $F_{2}=3.0$ (150 and $60 \mathrm{kt}$, respectively), and high stock of the top predator group at $F_{3}=10.0$ (200 kt). The carrying capacity increased linearly by 1980 from its constant value of 40 (2000 kt) up to 50 (2500 kt) in 1985 and retained this value until 1993. The $20 \%$ increase during the period of intense fishing represents the concomitantly higher resource availability from the lower trophic level in conjunction with nutrient enrichment and more intense plankton production in the Black Sea during the 1980s (c.f. Figs. 2 \& 3 in Oguz \& Gilbert 2007). This increase helped sustain the high stock regime of small pelagics somewhat longer during the 1980s at the time of most intense stock exploitation. Otherwise, the small pelagic stock would have started to decrease earlier and would not have been able to support the high catches observed during the 1980s. Starting in 1993, the carrying capacity reduced linearly, reaching its original value in 1999, in accordance with the decreasing trend in observed phytoplankton production.

Specification of harvesting rates. The temporal variation of small pelagic fishing mortality rate resembles that described by Daskalov et al. (in press) with some adjustments (Fig. 6). It attained fairly stable values of $f_{1} \approx 0.3$ until 1978; then it increased linearly, reaching 0.95 in 1986; and then declined, dropping steeply to 0.6 in 1990, and then more gradually to 0.3 in 1999 . Temporal variations of other fishing mortality rates were adjusted, in an idealized sense without introducing too many details, to the observed catch values. The medium pelagics fishing mortality rate retained a low steady value of 0.3 up to 1982 , and varied in a similar way to $f_{1}$ afterwards; it first increased up to 0.95 in 1989, then decreased to 0.4 in 1994 and kept this value for the rest of simulation period (Fig. 6). The fishing mortality rate of the top predator group ranged between 0.4 and 0.7 from 1964 to 1970 , and remained constant afterwards (Fig. 6). Setting $f_{3}=0.7$ allowed a relatively higher small pelagics stock prediction, as indicated by the equilibrium analysis (Fig. 5a), but the solutions were not especially sensitive to the exact figure, as long as it was above the threshold value of $f_{3} \approx 0.5$.
Temporal variation of stocks. The simulated stock and catch variations are depicted in Figs. $7 \& 8$, respectively. The small pelagics stock possessed 3 distinct successive transitions between its low regime $(<350 \mathrm{kt})$ and high regime (>950 kt) (Fig. 7a). The first transition (TR1) comprised the period from 1969 to 1973, during which the small pelagics stock switched to the high stock regime (HSR). Consistent with the former equilibrium analyses, the shift occurred at the large pelagics fishing mortality threshold rate of $f_{3} \approx$ 0.5 , at which the top predator stock dropped below 40 kt (Fig. 7c). Upon increasing $f_{3}$ to 0.7 , the top predator stock decreased further and vanished by the mid1970s, coincident with the transition of small pelagics from the low stock regime (LSR) to HSR (Fig. 4). The
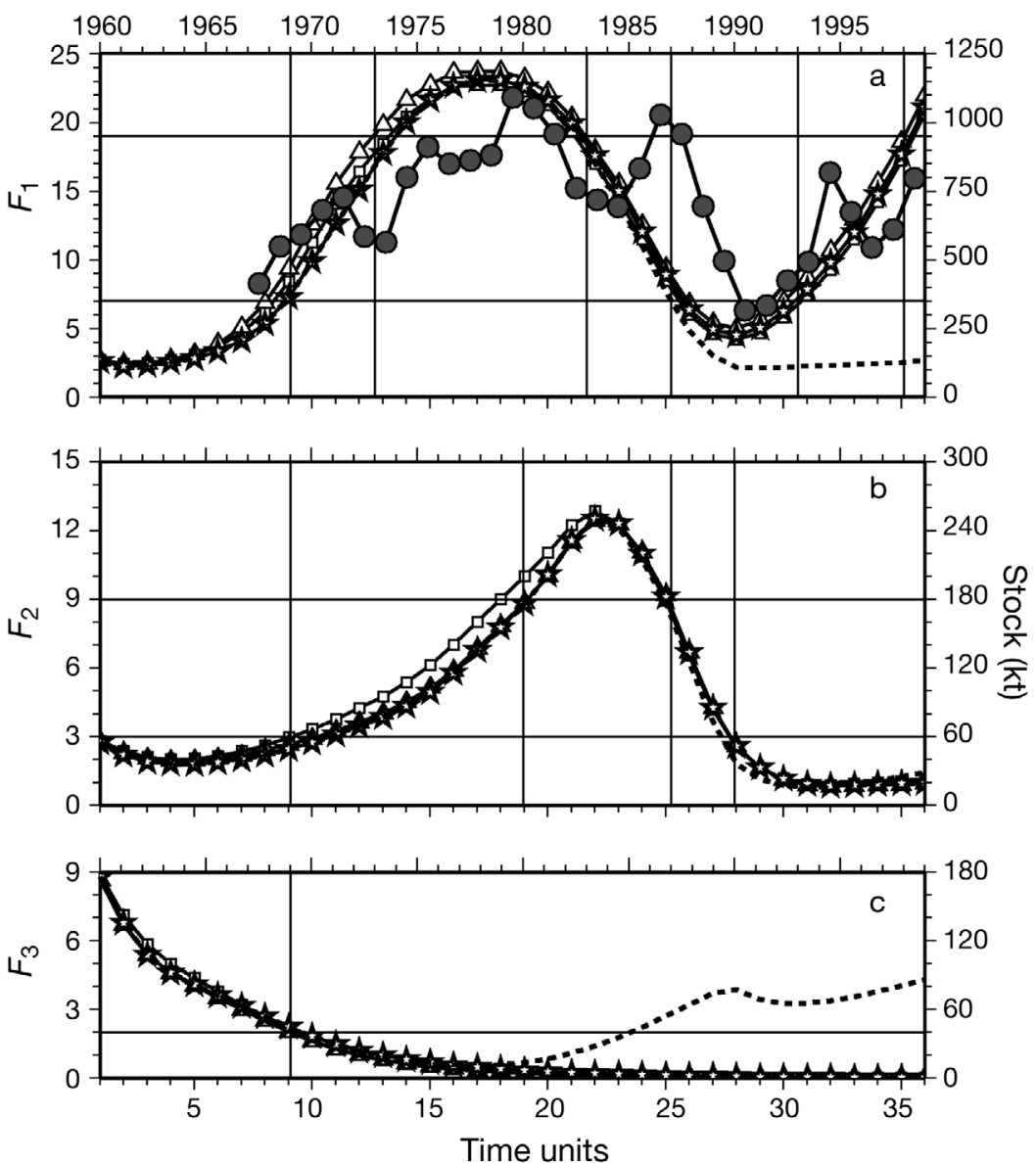

Fig. 7. Temporal variations of relative stock biomass for the (a) small pelagics group $\left(F_{1}\right)$, (b) medium pelagics group $\left(F_{2}\right)$, and (c) top predator group $\left(F_{3}\right)$ using the parameter values given in Table 2; $(\square)$ correspond to Simulation 1, $(\Delta)$ to Simulation 2 , and $(\star)$ to Simulation 3 . The dashed lines represent the results of the 4 th simulation using the alternative fishing mortality rate variations for the top predator group. The curve with $(O)$ in $(a)$ represents the small pelagics stock variation estimated by Daskalov et al. (2006). Horizontal lines are $(a, b)$ threshold of low and high stock regimes for the small and medium pelagic groups, respectively, and (c) threshold of low stock regime of the top predator group. Time periods between vertical lines identify either low or high stock regimes or the transitions between them 

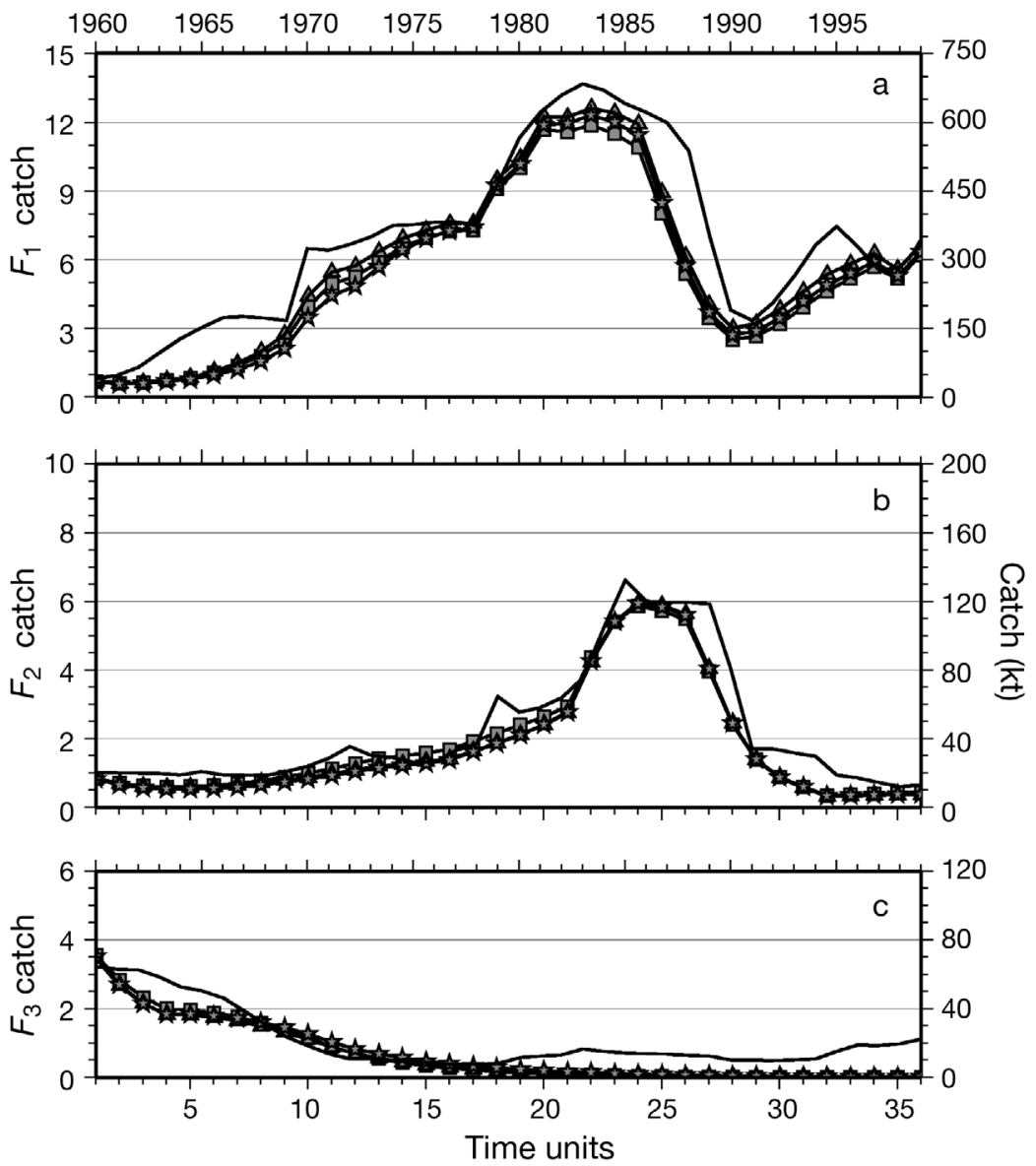

Fig. 8. Temporal variations of catches for the (a) small pelagics group, (b) medium pelagics group, (c) top predator group using the parameter values given in Table 2; the curves with $(\square)$ correspond to Simulation 1, $(\Delta)$ to Simulation 2, and $(\star)$ to Simulation 3. The curve without symbols represents the observed catch variations

harvesting rates of small and medium pelagics during the transition were $f_{1}=0.32$ to 0.35 and $f_{2}=0.3$, respectively.

The transition of medium pelagics stock from LSR ( $<60 \mathrm{kt}$ ) to HSR (>180 kt) followed that of small pelagics, with some time lag (Fig. 7b). It also started at the same threshold value of top fishing mortality rate $\left(f_{3} \approx\right.$ $0.5)$ during the late $1960 \mathrm{~s}$. It was initially a slow process from 1970 to 1975 at a time of rapid increase of small pelagics stock. Once this shift was completed, the medium pelagics stock alternated more rapidly between states during the second half of the 1970s.

The complementary steady-state analysis of the model, performed using the parameter values of the first simulation in Table 2, further elucidates dependence of TR1 on the harvesting rates of the small and medium pelagics groups. According to the equilibrium analysis (Fig. 9a), the small pelagics stock remained permanently in the low stable state for $f_{2}=0.2$ (not shown). The choices of $f_{2} \geq 0.4$, on the other hand, kept the medium pelagics permanently in the low stock state for all values of $f_{1}$ (Fig. 9b). The only favourable ranges of $f_{1}$ and $f_{2}$ that must accompany the $f_{3}$ threshold rate $\sim 0.55$ for the existence of TR1 of small and medium pelagics to their HSR appeared to be $f_{2}<0.4$ and $f_{1}$ $\leq 0.4$. The values of $f_{1}=0.32$ to 0.35 and $f_{2}=0.3$ used in the time-dependent simulations during TR1 were compatible with the ranges provided by the equilibrium analysis. These ranges of $f_{1}$ and $f_{2}$ will also alternate the medium pelagic stock to its high equilibrium state (Fig. 9b).

The HSR of small pelagics persisted until 1982, after which the second transition (TR2) between 1983 and 1986 brought the stock back to its LSR. TR2 initiated at $f_{1} \approx 0.6$, which again is in harmony with the equilibrium analysis (Curve 4 in Fig. 5a). Above this threshold value of $f_{1}$, the small pelagics stock was continually depleted at the expense of maintaining a steady catch level around $600 \mathrm{kt}$ (Fig. 8a). As the stock approached the LSR at higher values of $f_{1}$, the catch then dropped dramatically to $\sim 150 \mathrm{kt}$ within a few years.

Prior to their collapse, small pelagics were able to temporally support medium pelagics at their high stock level up to $250 \mathrm{kt}$ until 1985 under relatively low fishing mortality rates, around $f_{2} \approx 0.3$ to 0.4 (cf., Fig. 9b). Thereafter, as the fishing mortality rate $f_{2}$ exceeded 0.6 and food supply from small pelagics reached a critical level following the collapse of their stock, the medium pelagics stock started declining within the HSR, and finally switched back into the LSR between 1988 and 1989 concurrently with small pelagics (Fig. 7b). At $f_{2} \approx 0.6$, the catch became as high as $120 \mathrm{kt}$ and then dropped abruptly to less than $60 \mathrm{kt}$ (Fig. 8b) as the stock tended to approach the LSR. Thereafter, the medium pelagic stock remained within the LSR until the end of simulation period for a decreasing fishing mortality rate of 0.4 .

The LSR of small pelagics lasted for only $4 \mathrm{yr}$. Once its fishing mortality rate was reduced below the threshold value of 0.6 by 1993, the stock started increasing gradually (the third transition, TR3) and moved into the HSR by 1998 when $f_{1}<0.6$ and $f_{2} \sim 0.4$. As suggested by the equilibrium analysis (see the broken lines representing the return trajectories for decreasing $f_{1}$ in Fig. 9a,b) the crucial factor which promoted the switch was the specific choice of $f_{2}$ 

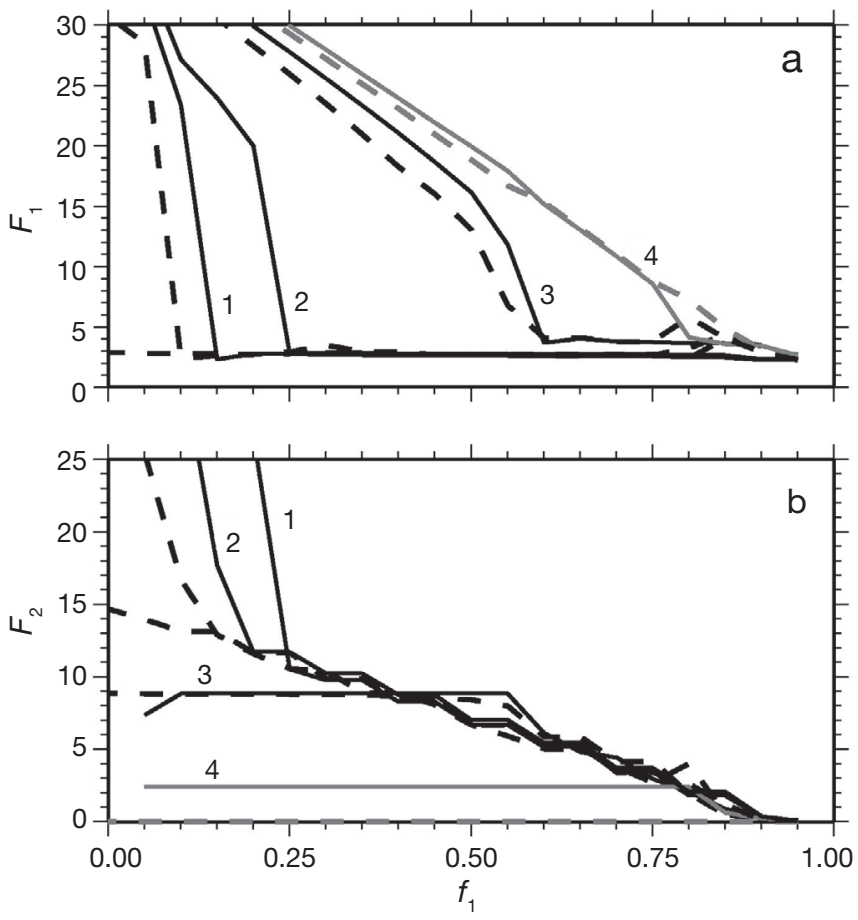

Fig. 9. Relative stock biomass $(F)$ versus small pelagics fishing mortality rate variations $\left(f_{1}\right)$ obtained from the equilibrium solution of the model for (a) small pelagics, (b) medium pelagics groups and the parameter values of Simulation 1 in Table 2 . The solutions are presented for $f_{3}=0.7$ and 4 different choices of the medium pelagic fishing mortality rate: $f_{2}=$ 0.25 (Curve 1), $f_{2}=0.28$ (Curve 2), $f_{2}=0.3$ (Curve 3), and $f_{2}=$ 0.4 (Curve 4). The continuous (broken) lines represent stock variations for increasing (decreasing) $f_{1}$

values during the transition. The values $f_{2} \approx 0.4$ supported the increase in $F_{1}$ when $0.45<f_{1}<0.60$ and kept $F_{2}$ depleted (Curve 4 in Fig. 9a,b), which was the case in the time-dependent simulations (Fig. 7b). The smaller values of $f_{2}$ shifted $F_{1}$ to the HSR at lower $f_{1}$ threshold values. It also shifted $F_{2}$ to HSR and thus would be a better stock recovery option for the small and medium pelagics stocks as further studied in the next subsection.

\section{Comparison with observations}

Bearing in mind the simplicity of the model, it reproduces adequately all major features of the multidecadal fish stock and catch variations. Contrary to a prolonged high stock phase up to 1987 in the empirical data (Fig. 7a), the modelled small pelagics stock, however, started decreasing by the early 1980s. One possible explanation for the difference is the composite representation of anchovy and sprat stocks in the model. According to the empirical data (cf. Figs. 11 \& 12 in Oguz et al. 2006), the anchovy stock declined by the early 1980s, whereas the sprat stock that is less commercially valuable and thus less exploited retained its high stock level somewhat longer. This difference is reflected in the total stock estimate data (Fig. $7 \mathrm{a}$ ) by a short-term peak during 1986-1987. Because the temporal variations in fishing pressure, $f_{1}$, were set primarily to that of anchovy in the model (since its catch was predominant), the modeled total small pelagics stock variations followed more closely those of the anchovy. Nevertheless, an extended HSR can be reproduced under somewhat lower fishing mortality rates of small pelagics. But, in this case, the total catch was underestimated by the model.

The difference in sprat and anchovy stock fluctuations may be partly related to climate-induced changes impacting temperature-dependent growth characteristics. Sprat is a cold water species spawning in autumn and winter months. Sprat abundance and growth are therefore expected to be more favourable during cold years, such as in the mid-1980s, known to be the coldest period of the previous century in the Black Sea (Oguz et al. 2006). A separate modelling of the anchovy and sprat stocks, together with their temperature-controlled growth parameterizations may provide a more realistic total small pelagics stock estimate.

The simulations predicted depletion of top predators by the mid-1970s, whereas a low level catch was always maintained in the empirical data (Fig. 8a). The difference likely arises due to an idealized (i.e. temporally uniform) specification of the top predator mortality rate $\left(f_{3}=0.7\right)$ until the end of simulation period. As depicted in Figs. $4 \mathrm{c} \& 5 \mathrm{c}$, setting $f_{3} \approx 0.5$ could be a more realistic choice but, in this case, the small pelagics stock and catch would be underestimated to some extent (cf. Fig. 5a). Nevertheless, the difference between the observed and computed catches is not critical in terms of model dynamics and does not alter the interpretations.

\section{Long-term stock variations under different harvesting conditions}

The previous simulations kept the top predator fishing mortality rate uniform at a value of 0.7 after 1970 . An interesting and notably different stocks organisation developed if the top predator fishing pressure was reduced after the depletion of its stock. The simulation described here reports the case when $f_{3}$ decreased from 0.7 in 1976 to 0.2 in 1981, retaining this value thereafter (see the broken lines in Fig. 6). The top predator stock then recovered partially to around $80 \mathrm{kt}$ (Fig. $7 \mathrm{c}$ ) at the expense of diminishing small and medium pelagics stocks (Fig. 7a,b). The system, therefore, changed drastically by reverting back to the 
pristine conditions. The increase in the top predator stock size under low $f_{3}$ was made possible by simultaneous decrease in $f_{1}$ from its high to moderate values and the low stock size of small pelagics. As described above, the equilibrium solution shown by Curve 1 in Fig. $5 \mathrm{c}$ is consistent with this case and suggests that a linear increase in $F_{3}$ up to $\sim 4$ (80 kt) is possible when $f_{3}=0.2, f_{2} \approx 0.2$ to 0.3 and $f_{1}$ decreases to $\sim 0.5$ from its higher values.

Interpretation of stock variations in terms of regime shifts

The time-dependent simulations performed by using 2 alternative settings of the top predator harvesting rate revealed considerably different stock progression events and associated regime shift dynamics. For the simulations of observed stock variations, the small pelagics stock $\left(F_{1}\right)$ and the top predator stock $\left(F_{3}\right)$ versus $f_{1}$ and $f_{3}$ plots (Fig. 10a,b) displayed a rapid rise of the small pelagics stock from its low to high stock regime (TR1) at constant $f_{1} \approx 0.3$ and an increasing $f_{3}$ from 0.4 to 0.7 , and vice versa for the top predator stock. TR2 occurred along constant $f_{3} \approx 0.7$ and for increasing $f_{1}$, as evident in the gradual decrease of $F_{1}$ and low values of $F_{3}$. The stocks were always characterized by a single equilibrium state on both sides of these threshold harvesting rates, and thus TR1 and TR2 represented smooth regime shifts. On the other hand, the simulation with the alternative top pelagics harvesting rate revealed multiple equilibria and alternative stable states for $0.2<f_{3}<0.7$ and $0.3<f_{1}<0.6$ for the small pelagics stock, and $0.3<f_{1}<0.9$ for the top pelagics stock (Fig. 11a,b). The low and high stock states thus alternated through the discontinuous regime shifts.

Future stock assessment scenarios

Motivated by the performance of the model in reproducing the long-term observed stock and catch variations, model simulations were further used to assess possible stock distributions under different harvesting conditions during the forthcoming $20 \mathrm{yr}$ (1999 to 2019). The parameter values were retained as in the hindcasting mode, implying that the internal conditions of the system would remain unchanged. Admittedly, this assumption may not be entirely realistic. The changes in life history traits, habitat alteration, changes in species assemblages, genetic changes (e.g. reduction in growth of overfished populations), as well as environmental conditions (e.g. resource availability, food competition with gelatinous species, climate) may alter the

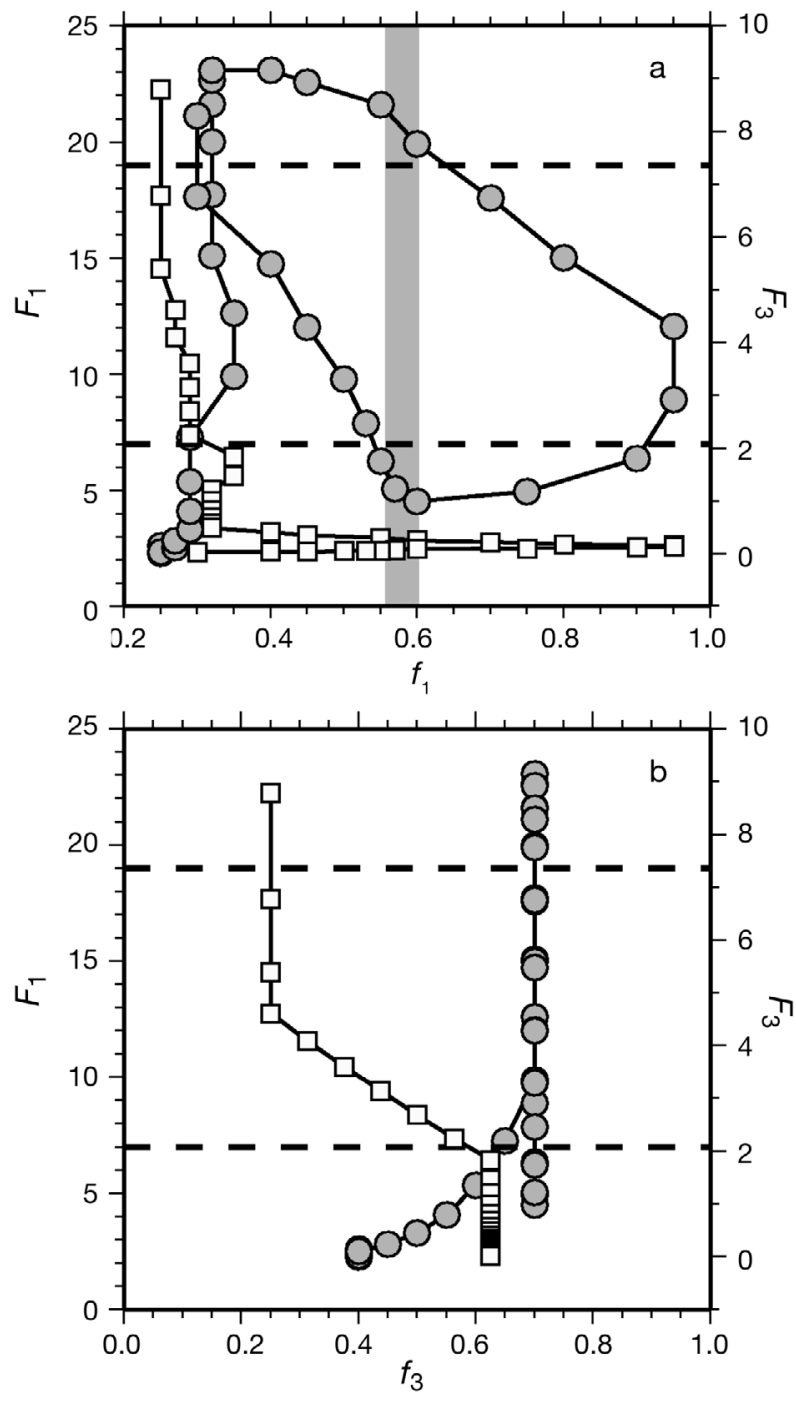

Fig. 10. Relative small pelagics stock biomass $\left(F_{1}, 0\right)$ and top predator stock biomass $\left(F_{3}, \square\right)$ versus the fishing mortality rate variations $\left(f_{1}, f_{3}\right)$ of (a) small pelagics group, (b) top predator group for Simulation 1 in Table 2. Broken lines represent the threshold values of stocks. Grey bar in panel (a) represents the threshold range of $f_{1}$ to switch small pelagic stock between high and low regimes

community dynamics and influence the recovery process (Conover \& Munch 2002, Hutchings \& Reynolds 2004). Nevertheless, the assessment of future stock variations under different harvesting scenarios alone may still be instructive for fishery management strategies that can mitigate or reverse stock depletion.

The first scenario of likely stock variations between 1999 and 2019 involves keeping the harvesting rates of small pelagics steady at 3 different values: $f_{1}=0.3,0.5$ and 0.7 , and keeping the other harvesting rates at 1990 s levels (i.e. $f_{2}=0.3, f_{3}=0.7$ ). The model integration started at 1960 and continued up to 2019. For $f_{1}=$ 0.3 , the small pelagics stock tended to increase up to 


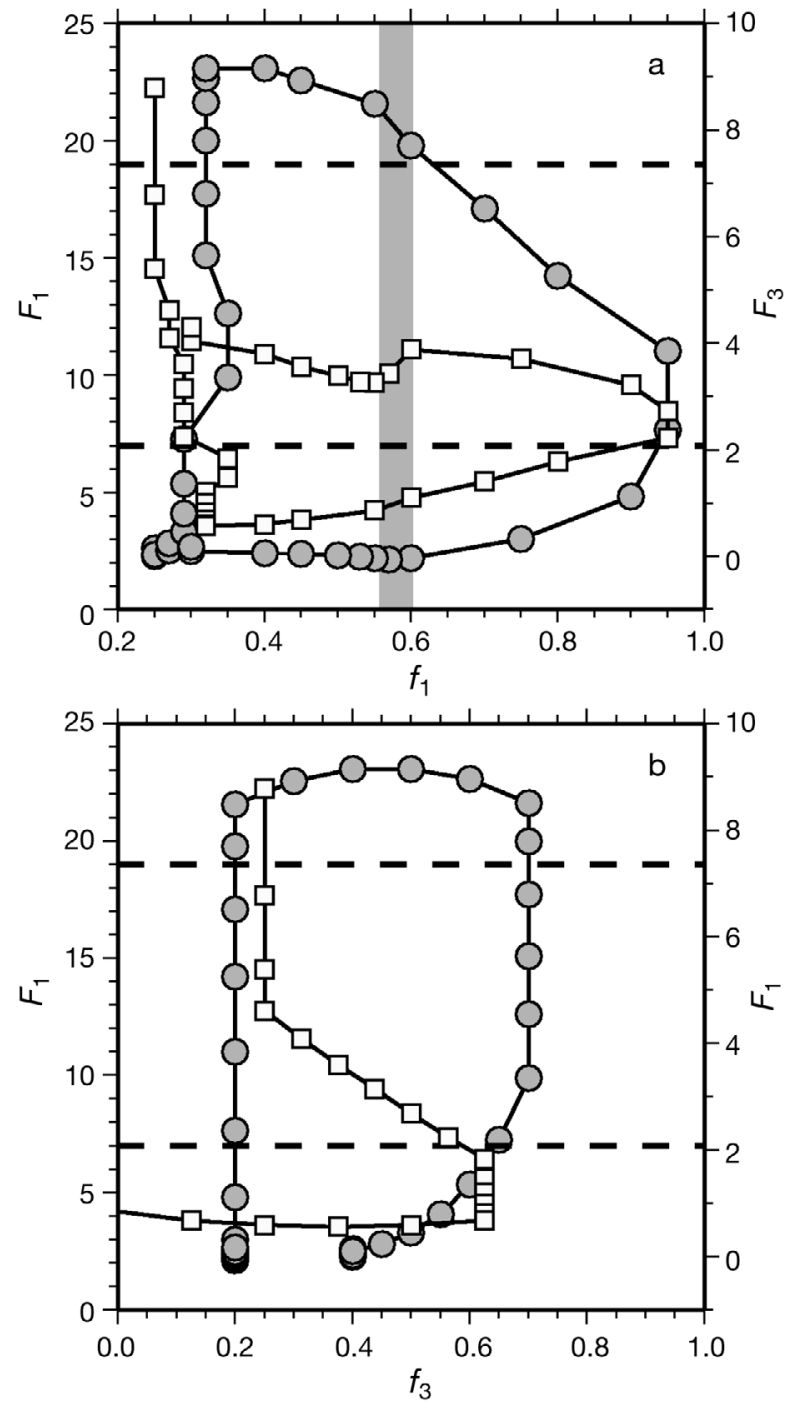

Fig. 11. Relative small pelagics stock biomass $\left(F_{1}, 0\right)$ and top predator stock biomass $\left(F_{3}, \square\right)$ versus the fishing mortality rate variations $\left(f_{1}, f_{3}\right)$ of (a) small pelagics group, (b) top predator group for the simulation with the alternative top predator fishing mortality rate variations shown in Fig. 6 by broken lines. Broken lines represent the threshold values of stocks. Grey bar in panel (a) represents the threshold range of $f_{1}$ to switch small pelagic stock between high and low regimes

$1500 \mathrm{kt}$ within the first $5 \mathrm{yr}$ and remained steady afterwards (Fig. 12a), implying its stabilisation in the HSR. The medium pelagics stock also recovered up to $150 \mathrm{kt}$ within the next 20 yr (Fig. 12b). Reduction in the harvesting rate of medium pelagics to 0.2 , on the other hand, helped building up their stock gradually to about 500 kt (Fig. 12b), with a compensatory reduction in the small pelagics stock to $1000 \mathrm{kt}$ (Fig. 12a). At this stage, both small and medium pelagics would be in their HSRs. Under this scenario, the top predator stocks would remain depleted and small pelagics would continue to play the main predator role in the

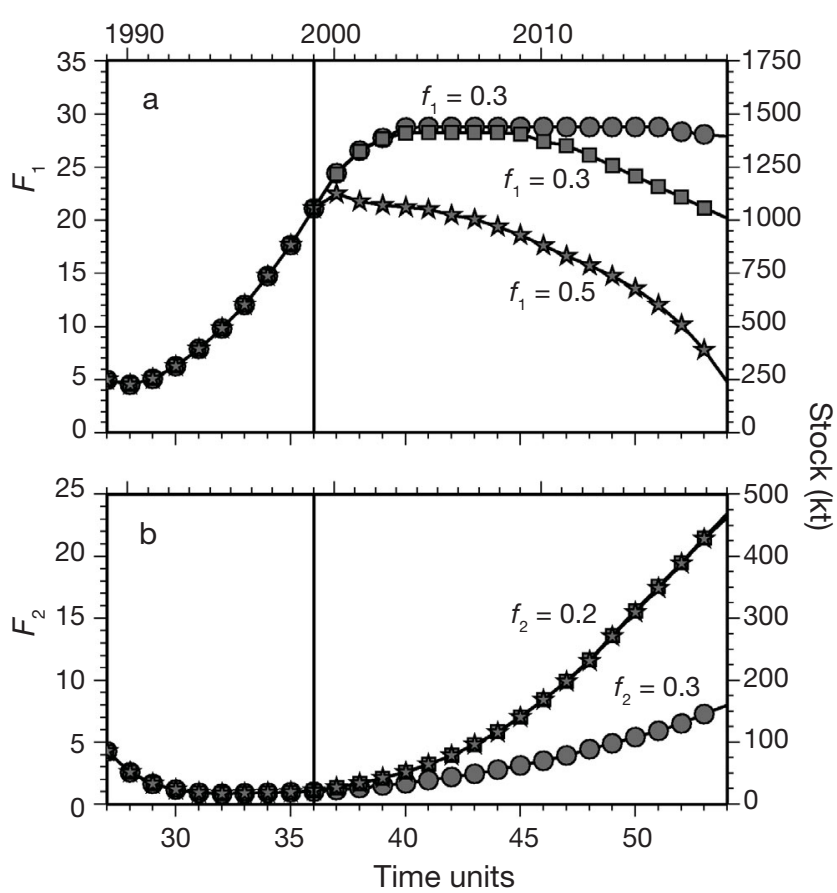

Fig. 12. Prediction of (a) small pelagics $\left(F_{1}\right)$, (b) medium pelagic stock biomass $\left(F_{2}\right)$ between 1999 and 2019 under 3 different settings of fishing mortality rate: $f_{1}=0.3$ and $f_{2}=0.3(0)$, $f_{1}=0.3$ and $f_{2}=0.2(\square)$, and $f_{1}=0.5$ and $f_{2}=0.2(\hbar)$. Vertical line represents the start of predictions in 1999

food web. Higher choice of $f_{1}$ (e.g. 0.5) would cause a continuous depletion of small pelagics stock from 1000 to $200 \mathrm{kt}$ within $20 \mathrm{yr}$ (Fig. 12a), while the medium pelagics stock increased linearly up to $500 \mathrm{kt}$ (Fig. 12b). The choices of $f_{1} \approx 0.3$ and $f_{2} \approx 0.25$ appears to be the most appropriate for keeping both groups in their high stock regime towards the end of next decade. The top predator stock may gradually build up if their harvesting rate reduces to 0.1 . This recovery will, however, take place at the expense of small pelagics stock and is not a desirable development; the preferred one is to have balanced stocks of small and medium pelagics while keeping the top predator stock at a relatively low level.

\section{DISCUSSION AND CONCLUSIONS}

Using a 3-trophic level predator-prey model, this study puts the long-term (1960 to 1999) Black Sea pelagic fish catch observations into a dynamical framework and simulates temporal variations of small, medium and large predatory pelagic fish stocks. The other issues addressed exploration of the dynamical response of the system to simultaneous changes in the harvesting rates and an assessment of their optimal conditions for sustainable yield of stocks. Understand- 
ing likely responses of the pelagic fish stocks to overexploitation constitutes an integral part of the efforts for elucidating the mechanisms that govern the longterm organisation of Black Sea ecosystem under different environmental factor combinations.

The equilibrium analyses suggested different stock arrangements for different combinations of the consumption and harvesting rates. The parameter values which best described the observed characteristics of stocks before and after 1970 were determined among these possible alternatives. The appropriate parameter values were then implemented in the time-dependent simulations to predict stock changes compatible with the available catch data. In essence, the modelling approach combined both steady-state and time dependent dynamics to arrive at a unified understanding of the mechanisms controlling the long-term fish stocks in the Black Sea. A thorough analysis of the equilibrium characteristics of the system under different parameter settings is useful for an understanding of many structural details of the stock progressions described by the time-dependent dynamics, and offers more realistic specification of harvesting rate variations in the time-dependent simulations.

The model analysis supports marked changes of the stocks after 1970 conjectured by the catch data. These changes were controlled primarily by simultaneous variations of the harvesting rates. The harvesting rate on small pelagics regulates all stocks through changes in resource availability (bottom-up control). Similarly, the harvesting rate on top pelagics changes the top predator stock size, which then alters the trophic cascade (top-down control). Their simultaneous changes introduce even more complex concurrently operating top-down and bottom-up controls within the higher trophic level and thus more complex dynamics of stock changes.

The pristine system prior to excessive harvesting of the stocks was dominated by a large predatory group whose sufficiently strong predation pressure maintained smaller species at their low population densities. This state of the system then shifted to the reverse configuration once the harvesting rate on the top predator group exceeded its critical threshold ( 0.5) and the harvesting rates on other groups were low $(\sim 0.3)$. The new state was populated by small and medium pelagics, whereas the top predator stock remained depleted. The system was disturbed subsequently by changes in harvesting pressure on small and medium pelagics. The small and medium pelagics stocks shifted simultaneously from their high to the low stock states when their harvesting rates exceeded the critical value of 0.6 . The small pelagics stock then shifted back again to the high stock state at the same threshold during the period of decreasing harvesting rate. The medium pelagics stock, however, stayed in the low stock regime with a harvesting rate of around 0.4 . The low stock regime of top predators remained unaltered after 1970, since their harvesting rates persisted above the threshold. All these stock configurations represent different forms of a single equilibrium state of the system and occur through smooth regime shifts. It was also shown that the top predator stock could shift back to the high stock state under a different threshold combination of harvesting rates (e.g. $f_{1}>$ 0.5 , and $f_{2}, f_{3} \leq 0.3$ ) and the system would then possess multiple equilibria and discontinuous regime shifts.

In addition to the changes in harvesting rates, changes in the consumption rate parameters $\left(r_{2}\right.$ and $\left.r_{3}\right)$ of the medium and top predator groups may also alternate the states. Their values, however, are not likely subject to large temporal variations and were therefore kept constant throughout the simulation period. The appropriate choice of their values is critical for the simulation of observed stock and catch variations. Their slightly different choices give rise to different stock arrangements which are inconsistent with the observations.

The Black Sea highest trophic level is characterized by a relatively simple structure (controlled by small pelagics) after the early 1970 s and represents a marked example of fishing down the food web effect. The present level of small pelagics stock is likely to be maintained if their harvesting rate is retained at around 0.5 during the next decade. The medium pelagics stock may, however, build up slowly if their harvesting rate is kept steady at around 0.2 to 0.3 . Building up of the top predator stock is even more challenging and requires maintaining their harvesting rate as low as 0.1 . But, in this case, the small pelagics stock must be reduced considerably, because the small pelagics and top predator groups can not co-exist at the same stable state under the present parameter setting. Considering the great economical value of anchovy for the region, the best management option seems to be sustaining balanced stocks of small and medium pelagics by harvesting them below their critical rates, while leaving the top predator stock depleted.

An important implication of the stock assessment simulations is the significance of a multi-species dynamical approach for fisheries management, due to the nonlinear responses of different stocks to the harvesting rates of different groups, as well as other environmental conditions. The classical single-species approach that ignores species and/or community interactions and maximizes the catch of a single target species/group would not be entirely adequate for managing sustainable utilization of future stocks. This assertion was tested by simplifying the model to the 
form of a prey-consumer system and a prey system alone and by prescribing the predator stocks from one of the simulations shown in Fig. 7. The reduced models were able to reproduce stock variations of the fullycoupled model only partially when the harvesting rate variations were adopted directly. The reconstruction of stock variations consistent with those provided by the full model was only possible when the harvesting rate variations were modified to some extent. The discrepancy between the harvesting rates of reduced and full models may be considered to reflect the difference between single and multi-species approach.

The present study highlights how a simple model, when carefully tuned, may provide detailed information on the dynamics of fish stocks and realistically reproduce the observations. In essence, the model dynamically reconstructs unobserved properties of the system by making use of a limited data set. It forms a basis for studying more complex systems involving anchovy and sprat populations as 2 independent resource groups, impacts of climate-induced fluctuations, and more proper representation of the enrichment effect (increasing carrying capacity) associated with intense eutrophication.

Acknowledgements. B. McKenzie and M. d'Alcala are thanked for their helpful comments on the manuscript. This study is a contribution to EU 6th Framework Programme Sesame Project (contract no: 036949-2), Turkish Scientific and Technological Research Council Project 104Y289, and NATO-CLG Project 982453.

\section{LITERATURE CITED}

Abrams PA, Roth JD (1994) The effects of enrichment of three-species food chains with nonlinear functional responses. Ecology 75:1118-1130

Bilio M, Niermann U (2004) Is the comb jelly really to blame for it all? Mnemiopsis leidyi and the ecological concerns about the Caspian Sea. Mar Ecol Prog Ser 269:173-183

Caswell H, Neubert MG (1998) Chaos and closure terms in plankton food chain models. J Plank Res 20:1837-1845

Chavez FP, Ryan J, Lluch-Cota SE, Niquen M (2003) From anchovies to sardines and back: multidecadal change in the Pacific Ocean. Science 299:217-221

Collie JS, Richardson K, Steele JH (2004) Regime shifts: can ecological theory illuminate the mechanisms? Prog Oceanogr 60:281-302

Conover DO, Munch SB (2002) Sustaining fisheries yields over evolutionary time scales. Science 297:94-96

Daskalov GM (2002) Overfishing drives a trophic cascade in the Black Sea. Mar Ecol Prog Ser 225:53-63

Daskalov GM (2003) Long-term changes in fish abundance and environmental indices in the Black Sea. Mar Ecol Prog Ser 255:259-270

Daskalov G, Prodanov K, Zengin M (in press) The Black Sea fisheries and ecosystem change: discriminating between natural variability and human-related effects. In: Nielsen J, Dodson J, Friedland K, Hamon T, Hughes N, Musick J,
Verspoor E (eds) Proceedings of the Fourth World Fisheries Congress: reconciling fisheries with conservation. American Fisheries Society, Symposium 49, Bethesda, $\mathrm{MD}, \mathrm{p}$ 587-602

de Young, B, Harris R, Alheit J, Beaugrand G, Mantua N, Shannon L (2004) Detecting regime shifts in the ocean: data considerations. Prog Oceanogr 60:143-164

Edwards MA, Yool A (2000) The role of higher predation in plankton population models. J Plank Res 22:1085-1112

Gibson GA, Musgrave DL, Hinckley S (2005) Non-linear dynamics of a pelagic ecosystem model with multiple predator and prey types. J Plank Res 27:427-447

Gücü AC (2002) Can overfishing be responsible for the successful establishment of Mnemiopsis leidyi in the Black Sea? Estuar Coast Shelf Sci 54:439-451

Hastings A, Powell T (1991) Chaos in a three-species food chain. Ecology 72:896-903

Hutchings JA (2000) Collapse and recovery of marine fishes. Nature 406:882-885

Hutchings JA, Reynolds JD (2004) Marine fish population collapses: consequences for recovery and extinction risk. Bioscience 54:297-309

Ivanov L, Panayotova M (2001) Determination of the Black Sea anchovy stocks during the period 1968 - 1993 by Ivanov's combined method. Proc Inst Oceanol Bulg Acad Sci 3:128-154

Jackson JB, Kirby MX, Berger WH, Bjorndal KA and 16 others (2001) Historical overfishing and the recent collapse of ecosystems. Science 293:629-638

Kemp WM, Brooks MT, Hood RR (2001) Nutrient enrichment, habitat variability and trophic transfer efficiency in simple models of pelagic ecosystems. Mar Ecol Prog Ser 223: 73-87

Kideys AE (2002) Fall and rise of the Black Sea ecosystem. Science 297:1482-1484

Lima ID, Olson DB, Doney SC (2002) Intrinsic dynamics and stability properties of size-structured pelagic ecosystem models. J Plank Res 24:533-556

May RM (1977) Thresholds and breakpoints in ecosystems with multiplicity of stable states. Nature 269:471-477

May RM, Beddington JR, Clark JW, Sidney JH, Laws RM (1979) Management of multispecies fisheries. Science 205: 267-277

Morozov AY, Nezlin NP, Petrovskii SV (2005) Invasion of a top predator into an epipelagic ecosystem can bring a paradoxical top-down trophic control. Biol Invasions 7: 845-861

Myers RA, Worm B (2003) Rapid worldwide depletion of predatory fish communities. Nature 423:280-283

Oguz T (2005a) Long term impacts of anthropogenic forcing on the reorganisation of the Black Sea ecosystem. Oceanography 18:112-121

Oguz T (2005b) Black Sea ecosystem response to climatic variations. Oceanography 18:122-133

Oguz T, Gilbert D (2007) Abrupt transitions of the top-down controlled Black Sea pelagic ecosystem during 19602000: evidence for regime shifts under strong fishery exploitation and nutrient enrichment modulated by climate-induced variations. Deep-Sea Res I 54:220-242, doi:10.1016/j.dsr.2006.09.010

Oguz T, Dippner JW, Kaymaz Z (2006) Climatic regulation of the Black Sea hydro-meteorological and ecological properties at interannual-to-decadal time scales. J Mar Syst 60: 235-254

Pauly D, Christensen V, Dallsgaard J, Froese R, Torres F (1998) Fishing down marine food webs. Science 279: 860-863 
Petraitis PS, Dudgeon RD (2004) Detection of alternative stable states in marine communities. J Exp Mar Biol Ecol 300: 343-371

Scheffer M, Carpenter S (2003) Regime-shifts in ecosystems: models and evidence. Trend Ecol Evol 18:648-656

Scheffer M, Rinaldi S, Kuznetsov YA (2000) Effects of fish on plankton dynamics: a theoretical analysis. Can J Fish Aquat Sci 57:1208-1219

Scheffer M, Carpenter S, Folley JA, Folke C, Walker B (2001) Catastrophic shifts in ecosystems. Nature 413:591-596

Spencer PD, Collie JS (1995) A simple predator-prey model of exploited marine fish populations incorporating alternative prey. ICES J Mar Sci 53:615-628

Steele JH, Henderson EW (1984) Modeling long-term

Editorial responsibility: Howard Browman (Associate Editorin-Chief), Storebø, Norway fluctuations in fish stocks. Science 224:985-986

Yel M, Ozdamar E, Amaha A, Miyazaki N (1996) Some aspects of the dolphin fishery on the Turkish coast of the Black Sea. In: Ozturk (ed) Proceedings 1st International Symposium on Marine Mammals of the Black Sea. ACAR Matbaacilik AS, Istanbul, p 31-40

Zaitsev Y, Mamaev V (1997) Marine biological diversity in the Black Sea: a study of change and decline. Black Sea Environmental Series, Vol 3. United Nations Publications, New York

Zemsky VA (1996) History of Russian fishery of dolphins in the Black Sea. In: Öztürk (ed) Proceedings 1st International Symposium on Marine Mammals of the Black Sea. ACAR Matbaacilik AS, Istanbul, p 46-48

Submitted: September 25, 2006; Accepted: March 27, 2007 Proofs received from author(s): August 24, 2007 